SUPPORTING INFORMATION

\title{
Hydrogen Transfer to Ketones Catalyzed by the Shvo's Ruthenium Hydride Complex: A Mechanistic Insight
}

Aleix Comas-Vives, Gregori Ujaque* and Agustí Lledós*

Unitat de Química Física, Departament de Química, Edifici Cn, Universitat Autònoma de Barcelona, 08193 Bellaterra, Catalonia, Spain

\section{Contents}

1. Complete Gaussian Cite

2. Absolute Energies and Cartesian Coordinates of the Optimized Structures

2.1. Gaussian Calculations

2.2. Jaguar Calculations

3. Comparison between B3LYP, MP2 and CCSD(T) calculations for the model system. 


\section{Complete Gaussian Cite}

Gaussian 03, Revision C.02, M. J. Frisch, G. W. Trucks, H. B. Schlegel, G. E. Scuseria, M. A. Robb, J. R. Cheeseman, J. A. Montgomery, Jr., T. Vreven, K. N. Kudin, J. C. Burant, J. M. Millam, S. S. Iyengar, J. Tomasi, V. Barone, B. Mennucci, M. Cossi, G. Scalmani, N. Rega, G. A. Petersson, H. Nakatsuji, M. Hada, M. Ehara, K. Toyota, R. Fukuda, J. Hasegawa, M. Ishida, T. Nakajima, Y. Honda, O. Kitao, H. Nakai, M. Klene, X. Li, J. E. Knox, H. P. Hratchian, J. B. Cross, C. Adamo, J. Jaramillo, R. Gomperts, R. E. Stratmann, O. Yazyev, A. J. Austin, R. Cammi, C. Pomelli, J. W. Ochterski, P. Y. Ayala, K. Morokuma, G. A. Voth, P. Salvador, J. J. Dannenberg, V. G. Zakrzewski, S. Dapprich, A. D. Daniels, M. C. Strain, O. Farkas, D. K. Malick, A. D. Rabuck, K. Raghavachari, J. B. Foresman, J. V. Ortiz, Q. Cui, A. G. Baboul, S. Clifford, J. Cioslowski, B. B. Stefanov, G. Liu, A. Liashenko, P. Piskorz, I. Komaromi, R. L. Martin, D. J. Fox, T. Keith, M. A. Al-Laham, C. Y. Peng, A. Nanayakkara, M. Challacombe, P. M. W. Gill, B. Johnson, W. Chen, M. W. Wong, C. Gonzalez and J. A. Pople, Gaussian, Inc., Wallingford CT, 2004.

\section{Absolute Energies and Cartesian Coordinates of the Optimized Structures}

\subsection{Gaussian Calculations}

a1

$$
\begin{array}{ll}
E(B 3 L Y P)=-589.954236256 & E(C P C M)=-589.952536 \\
E(M P 2))=-587.934419360 & E(C C S D(T))=-588.0115917
\end{array}
$$

$\begin{array}{cccc}44 & 0.397310 & -0.066747 & -0.250364 \\ 1 & 1.072103 & -0.058885 & -1.693164 \\ 6 & -1.798391 & -0.126875 & -0.980487 \\ 6 & -1.245326 & -1.450825 & -1.026006 \\ 6 & -0.962390 & -1.849158 & 0.307810 \\ 6 & -1.378317 & -0.803651 & 1.202110 \\ 6 & -1.930571 & 0.230274 & 0.403903 \\ 1 & -2.167298 & 0.443474 & -1.822974 \\ 1 & -1.072911 & -2.032572 & -1.920333 \\ 1 & -0.542701 & -2.800001 & 0.608151 \\ 1 & -1.340655 & -0.812952 & 2.281919 \\ 6 & 2.058258 & -0.722779 & 0.363965\end{array}$




$\begin{array}{rrrr}8 & 3.066391 & -1.152276 & 0.731567 \\ 8 & -2.501040 & 1.339583 & 0.922264 \\ 1 & -2.755325 & 1.936906 & 0.206656 \\ 6 & 0.982791 & 1.730831 & -0.136645 \\ 8 & 1.305754 & 2.839440 & -0.085348\end{array}$

a2

$$
\mathrm{E}(\mathrm{B} 3 \mathrm{LYP})=-589.953876186
$$

$$
E(M P 2)=-587.934275986
$$$$
G=-589.878513
$$$$
\mathrm{G}(\mathrm{OD})=-589.882083
$$

$\begin{array}{crrr}44 & 0.344975 & -0.000818 & -0.106425 \\ 1 & 0.484471 & 0.027338 & -1.699609 \\ 6 & -1.623424 & -1.170890 & 0.278807 \\ 6 & -1.074349 & -0.781170 & 1.558248 \\ 6 & -1.080725 & 0.627967 & 1.629124 \\ 6 & -1.626722 & 1.138300 & 0.392757 \\ 6 & -2.039991 & 0.021371 & -0.386073 \\ 1 & -1.815170 & -2.183255 & -0.052371 \\ 1 & -0.721790 & -1.461492 & 2.321496 \\ 1 & -0.731851 & 1.228699 & 2.457825 \\ 1 & -1.816204 & 2.173025 & 0.144200 \\ 6 & 1.628385 & 1.384048 & -0.143332 \\ 8 & 2.389803 & 2.252708 & -0.170580 \\ 6 & 1.671337 & -1.342515 & -0.170368 \\ 8 & 2.459854 & -2.186601 & -0.214311 \\ 8 & -2.724782 & 0.144417 & -1.542987 \\ 1 & -2.704440 & -0.695171 & -2.020811\end{array}$

$\mathbf{H}_{\mathbf{2}} \mathbf{C}=\mathbf{O} \quad \mathrm{E}(\mathrm{B} 3 \mathrm{LYP})=-114.500879906$

$$
\mathrm{E}(\mathrm{MP} 2)=-114.17899135286
$$

$$
G=-114.495813
$$

$$
\begin{array}{rrrr}
6 & -0.000001 & -0.529213 & 0.000000 \\
8 & -0.000001 & 0.677608 & 0.000000 \\
1 & 0.000005 & -1.122791 & 0.938338 \\
1 & 0.000005 & -1.122791 & -0.938338
\end{array}
$$

$\mathrm{E}(\mathrm{CPCM})=-589.952197$

$\mathrm{E}(\operatorname{CCSD}(\mathrm{T}))=-588.01182880$

$\mathrm{G}(\mathrm{CD})=-589.880898$

$\mathrm{G}(\mathrm{CD}-\mathrm{OD})=-589.884467$
$\mathrm{E}(\mathrm{CPCM})=-114.501484$

$\mathrm{E}(\operatorname{CCSD}(\mathrm{T}))=-114.2030076$
$\mathbf{C H}_{3} \mathbf{O H} \quad \mathrm{E}(\mathrm{B} 3 \mathrm{LYP})=-115.721857587$
$\mathrm{E}(\mathrm{CPCM})=-115.72236$
$\mathrm{E}(\mathrm{MP} 2)=-115.377996835$
$\mathrm{E}(\operatorname{CCSD}(\mathrm{T}))=-115.4084246$ 


$\begin{array}{rrrr}1 & 1.038792 & -0.540912 & 0.892890 \\ 1 & -1.134979 & -0.762354 & 0.001054 \\ 6 & 0.661273 & -0.019499 & -0.000096 \\ 8 & -0.748959 & 0.121987 & -0.000105 \\ 1 & 1.082159 & 0.989502 & -0.001748 \\ 1 & 1.038059 & -0.545138 & -0.890783\end{array}$

$1 a$

$$
\begin{aligned}
& E(B 3 L Y P)=-476.565683710 \\
& E(M P 2)=-474.81440697033
\end{aligned}
$$$$
\begin{array}{llll}
44 & -0.451703 & -0.346685 & -0.305107
\end{array}
$$$$
\begin{array}{llll}
1 & -1.035237 & -0.497809 & -1.794451
\end{array}
$$$$
\begin{array}{llll}
6 & 1.604344 & 0.501941 & -0.942377
\end{array}
$$$$
\begin{array}{llll}
6 & 0.591209 & 1.493346 & -0.717024
\end{array}
$$$$
\begin{array}{llll}
6 & 0.268274 & 1.467113 & 0.695142
\end{array}
$$$$
\begin{array}{llll}
6 & 1.080853 & 0.488700 & 1.338205
\end{array}
$$$$
\begin{array}{llll}
6 & 1.894994 & -0.089873 & 0.322790
\end{array}
$$$$
\begin{array}{llll}
1 & 2.100207 & 0.285030 & -1.879053
\end{array}
$$$$
\begin{array}{llll}
1 & 0.218162 & 2.200551 & -1.444383
\end{array}
$$$$
\begin{array}{llll}
1 & -0.424928 & 2.135294 & 1.188563
\end{array}
$$$$
\begin{array}{llll}
1 & 1.105877 & 0.232719 & 2.387095
\end{array}
$$$$
\begin{array}{llll}
6 & -2.236123 & -0.242176 & 0.254227
\end{array}
$$$$
\begin{array}{llll}
8 & -3.331791 & -0.098408 & 0.610007
\end{array}
$$$$
\begin{array}{llll}
8 & 2.768169 & -1.084130 & 0.578132
\end{array}
$$$$
1 \quad 3.198525 \quad-1.355664 \quad-0.243939
$$

$$
\begin{array}{lll}
\mathrm{E}(\mathrm{B} 3 \mathrm{LYP})=-113.306913781 & \mathrm{E}(\mathrm{CPCM})=-113.303638 \\
\mathrm{E}(\mathrm{MP} 2)=-113.01620666967 & \mathrm{E}(\mathrm{CCSD}(\mathrm{T}))=-113.0335137
\end{array}
$$$$
\begin{array}{llll}
6 & 0.000000 & 0.000000 & -0.650127
\end{array}
$$$$
\begin{array}{llll}
8 & 0.000000 & 0.000000 & 0.487595
\end{array}
$$

$$
\text { 2a } \begin{array}{ll}
\mathrm{E}(\mathrm{B} 3 \mathrm{LYP})=-591.110324082 & \mathrm{E}(\mathrm{CPCM})=-591.10773 \\
\mathrm{E}(\mathrm{MP} 2)=-589.06496827034 & \mathrm{E}(\mathrm{CCSD}(\mathrm{T}))=-589.1662125
\end{array}
$$

\author{
$\mathrm{E}(\mathrm{CPCM})=-476.576099$ \\ $\mathrm{E}(\operatorname{CCSD}(\mathrm{T}))=-474.8931441$
}




$\begin{array}{rrrr}1 & 0.202420 & -2.896788 & -0.512496 \\ 1 & 1.033305 & -1.006792 & -2.284432 \\ 6 & -2.130094 & -0.415060 & -0.344781 \\ 8 & -3.197398 & -0.685218 & -0.697163 \\ 8 & 2.298211 & 1.205557 & -0.956112 \\ 1 & 1.519203 & 1.802676 & -1.029891 \\ 6 & -0.600511 & 2.064663 & 0.783306 \\ 8 & -0.360946 & 1.953548 & -0.497016 \\ 1 & -1.615536 & 2.306325 & 1.108039 \\ 1 & 0.208633 & 2.375394 & 1.453620\end{array}$

$\begin{array}{lll}\text { ts1a } & \mathrm{E}(\mathrm{B} 3 \mathrm{LYP})=-591.097648012 & \mathrm{E}(\mathrm{CPCM})=-591.096312 \\ \mathrm{E}(\mathrm{MP} 2)=-589.04069689450 & \mathrm{E}(\mathrm{CCSD}(\mathrm{T})=-589.1515337\end{array}$

1 Imaginary Frequency $=-721.3 \mathrm{~cm}^{-1}$

$\begin{array}{cccc}44 & -0.351555 & 0.005103 & 0.177747 \\ 1 & -1.034778 & 0.812582 & 1.479956 \\ 6 & 1.798671 & -0.263365 & 0.964995 \\ 6 & 1.056836 & -1.469036 & 1.194493 \\ 6 & 0.654636 & -1.977711 & -0.080259 \\ 6 & 1.150084 & -1.101367 & -1.105257 \\ 6 & 1.848816 & -0.037537 & -0.442371 \\ 1 & 2.238686 & 0.380902 & 1.714483 \\ 1 & 0.866843 & -1.932989 & 2.152090 \\ 1 & 0.106850 & -2.895819 & -0.247154 \\ 1 & 1.041832 & -1.215276 & -2.174319 \\ 6 & -2.089421 & -0.517487 & -0.336725 \\ 8 & -3.126580 & -0.912984 & -0.666832 \\ 8 & 2.361615 & 1.068844 & -1.042747 \\ 1 & 1.640739 & 1.731155 & -1.067079 \\ 6 & -0.788045 & 2.133609 & 0.747470 \\ 8 & -0.395361 & 2.005958 & -0.506248 \\ 1 & -1.824116 & 2.453281 & 0.919269 \\ 1 & -0.074482 & 2.544435 & 1.474409\end{array}$

$3 a$

$$
\begin{array}{ll}
\mathrm{E}(\mathrm{B} 3 \mathrm{LYP})=-591.101263668 & \mathrm{E}(\mathrm{CPCM})=-591.100576 \\
\mathrm{E}(\mathrm{MP} 2)=-589.04170246939 & \mathrm{E}(\mathrm{CCSD}(\mathrm{T}))=-589.1577037
\end{array}
$$

$\begin{array}{cccc}44 & -0.338864 & 0.001638 & 0.184961 \\ 1 & -0.937648 & 1.311851 & 1.417081 \\ 6 & 1.810894 & -0.341727 & 0.941812 \\ 6 & 1.031673 & -1.529094 & 1.181571 \\ 6 & 0.570336 & -1.998989 & -0.082690 \\ 6 & 1.044115 & -1.103554 & -1.110551 \\ 6 & 1.831388 & -0.085898 & -0.455169\end{array}$




$\begin{array}{rrrr}1 & 2.278251 & 0.284015 & 1.691208 \\ 1 & 0.865281 & -2.004159 & 2.138107 \\ 1 & -0.013818 & -2.894347 & -0.250056 \\ 1 & 0.898954 & -1.196926 & -2.177443 \\ 6 & -2.098382 & -0.492680 & -0.297419 \\ 8 & -3.135033 & -0.896483 & -0.620631 \\ 8 & 2.321604 & 1.030411 & -1.045851 \\ 1 & 1.580939 & 1.684288 & -1.030622 \\ 6 & -0.720170 & 2.284808 & 0.734281 \\ 8 & -0.297823 & 1.959139 & -0.523710 \\ 1 & -1.704473 & 2.775785 & 0.772927 \\ 1 & 0.013417 & 2.825679 & 1.351040\end{array}$

$4 a 2$

$$
\begin{array}{ll}
\mathrm{E}(\mathrm{B} 3 \mathrm{LYP})=-704.461378941 & \mathrm{E}(\mathrm{CPCM})=-704.461203 \\
\mathrm{E}(\mathrm{MP} 2)=-702.11563302069 & \mathrm{E}(\mathrm{CCSD}(\mathrm{T}))=-702.227153
\end{array}
$$

$\begin{array}{cccc}44 & -0.199142 & 0.053302 & 0.021602 \\ 1 & -2.282787 & -2.181467 & -0.770941 \\ 6 & 1.690867 & -0.645701 & 1.232675 \\ 6 & 0.892242 & -1.795829 & 0.867763 \\ 6 & 0.901466 & -1.904935 & -0.542654 \\ 6 & 1.663015 & -0.821698 & -1.082171 \\ 6 & 2.212789 & -0.099468 & 0.028080 \\ 1 & 1.932054 & -0.324598 & 2.237868 \\ 1 & 0.410747 & -2.471963 & 1.560999 \\ 1 & 0.355128 & -2.632936 & -1.126239 \\ 1 & 1.877079 & -0.630418 & -2.123749 \\ 8 & -1.586018 & -0.315929 & -1.455613 \\ 6 & -1.466002 & 0.304356 & 1.403113 \\ 8 & -2.180428 & 0.448570 & 2.300076 \\ 1 & 3.255494 & 1.332939 & 0.729610 \\ 8 & 3.080761 & 0.920391 & -0.126990 \\ 6 & -2.623143 & -1.194318 & -1.141014 \\ 1 & -3.328899 & -0.793813 & -0.389612 \\ 1 & -3.201577 & -1.378543 & -2.060970 \\ 6 & -0.302824 & 1.884556 & -0.490004 \\ 8 & -0.322496 & 2.993687 & -0.800244\end{array}$

$\begin{array}{cccc}\text { ts2a } & \mathrm{E}(\mathrm{B} 3 \mathrm{LYP})=-704.456006417 & \mathrm{E}(\mathrm{CPCM})=-704.455717 \\ & \mathrm{E}(\mathrm{MP} 2)=-702.11094196316 & \mathrm{E}(\mathrm{CCSD}(\mathrm{T}))=-702.2209248 \\ & & & \\ & 1 \text { Imaginary Frequency }=-42.9 \mathrm{~cm}^{-1} & \\ 44 & 0.234653 & 0.072202 & -0.128082 \\ 1 & 0.907750 & -2.289477 & 1.843399 \\ 6 & -1.864755 & 0.583453 & -1.049734\end{array}$




$\begin{array}{rrrr}6 & -1.226168 & -0.398880 & -1.860654 \\ 6 & -1.101307 & -1.597605 & -1.073924 \\ 6 & -1.649669 & -1.344500 & 0.208101 \\ 6 & -2.132144 & -0.002397 & 0.231806 \\ 1 & -2.125943 & 1.589174 & -1.355423 \\ 1 & -0.937431 & -0.276533 & -2.895221 \\ 1 & -0.680836 & -2.536101 & -1.408957 \\ 1 & -1.666612 & -2.018922 & 1.052841 \\ 8 & 0.778735 & -0.188507 & 1.839937 \\ 6 & 1.901107 & -0.445289 & -0.862074 \\ 8 & 2.879428 & -0.769795 & -1.384137 \\ 1 & -2.827853 & 1.473235 & 1.231615 \\ 8 & -2.829139 & 0.508419 & 1.275733 \\ 6 & 1.445005 & -1.373109 & 2.155829 \\ 1 & 2.463379 & -1.432923 & 1.729180 \\ 1 & 1.549976 & -1.419061 & 3.251853 \\ 6 & 0.823041 & 1.856084 & 0.075128 \\ 8 & 1.148750 & 2.958282 & 0.173396\end{array}$

$5 a$

$$
\begin{array}{ll}
\mathrm{E}(\mathrm{B} 3 \mathrm{LYP})=-704.492705483 & \mathrm{E}(\mathrm{CPCM})=-704.488541 \\
\mathrm{E}(\mathrm{MP} 2)=-702.16199556120 & \mathrm{E}(\mathrm{CCSD}(\mathrm{T}))=-702.2595747
\end{array}
$$

$\begin{array}{crrr}44 & 0.296813 & -0.200027 & 0.008810 \\ 1 & -0.147818 & 3.188061 & 0.759433 \\ 6 & -1.585906 & -1.140215 & -0.850796 \\ 6 & -0.912944 & -2.084992 & -0.023120 \\ 6 & -0.894346 & -1.573758 & 1.317402 \\ 6 & -1.558282 & -0.312322 & 1.324045 \\ 6 & -2.151130 & -0.065793 & -0.006618 \\ 1 & -1.790283 & -1.248934 & -1.907934 \\ 1 & -0.504132 & -3.038256 & -0.332721 \\ 1 & -0.470778 & -2.083669 & 2.173109 \\ 1 & -1.742662 & 0.305535 & 2.193222 \\ 8 & -0.258756 & 1.848300 & -0.855232 \\ 6 & 1.642005 & 0.265488 & 1.286570 \\ 8 & 2.416932 & 0.495330 & 2.111961 \\ 1 & -1.250500 & 1.785928 & -0.815613 \\ 8 & -2.745117 & 0.964293 & -0.392733 \\ 6 & 0.165744 & 3.092121 & -0.286721 \\ 1 & 1.254701 & 3.121547 & -0.342182 \\ 1 & -0.242974 & 3.925626 & -0.867888 \\ 6 & 1.595337 & -0.697557 & -1.308392 \\ 8 & 2.340918 & -1.064484 & -2.109152\end{array}$

b

$$
\begin{array}{ll}
\mathrm{E}(\mathrm{B} 3 \mathrm{LYP})=-588.733267359 & \mathrm{E}(\mathrm{CPCM})=-588.73726 \\
\mathrm{E}(\mathrm{MP} 2)=-586.73913217903 & \mathrm{E}(\mathrm{CCSD}(\mathrm{T})=-586.8013338
\end{array}
$$




$\begin{array}{cccc}44 & 0.306804 & 0.000000 & -0.185905 \\ 6 & 1.623803 & 1.394038 & -0.207745 \\ 8 & 2.378538 & 2.263134 & -0.131586 \\ 6 & 1.623903 & -1.393943 & -0.207855 \\ 8 & 2.378732 & -2.262956 & -0.131684 \\ 6 & -1.588212 & -1.170974 & 0.319855 \\ 6 & -0.953460 & -0.725089 & 1.497323 \\ 6 & -0.953452 & 0.724739 & 1.497471 \\ 6 & -1.588197 & 1.170874 & 0.320095 \\ 6 & -2.185219 & 0.000026 & -0.388910 \\ 1 & -1.770279 & -2.200700 & 0.041318 \\ 1 & -0.549531 & -1.341541 & 2.290350 \\ 1 & -0.549508 & 1.341020 & 2.290624 \\ 1 & -1.770283 & 2.200659 & 0.041787 \\ 8 & -2.849117 & 0.000136 & -1.419940\end{array}$

$1 b$

$$
\begin{array}{ll}
\mathrm{E}(\mathrm{B} 3 \mathrm{LYP})=-704.471607126 & \mathrm{E}(\mathrm{CPCM})=-704.465314 \\
\mathrm{E}(\mathrm{MP} 2)=-702.13330746864 & \mathrm{E}(\mathrm{CCSD}(\mathrm{T}))=-702.23349651
\end{array}
$$
ts1b $\quad \mathrm{E}(\mathrm{B} 3 \mathrm{LYP})=-704.423961288 \quad \mathrm{E}(\mathrm{CPCM})=-704.422275$
$\mathrm{E}(\mathrm{MP} 2)=-702.07366295184 \quad \mathrm{E}(\mathrm{CCSD}(\mathrm{T}))=-702.18335443$
1 Imaginary Frequency $=-98.6 \mathrm{~cm}^{-1}$




$\begin{array}{cccc}44 & 0.592017 & -0.092302 & -0.143682 \\ 1 & 0.851005 & 0.011377 & -1.704438 \\ 6 & -1.164835 & -1.593408 & -0.130564 \\ 6 & -0.764510 & -1.427207 & 1.249052 \\ 6 & -1.468684 & -0.324846 & 1.771148 \\ 6 & -2.410350 & 0.126124 & 0.796477 \\ 6 & -2.231540 & -0.650642 & -0.350580 \\ 1 & -1.016558 & -2.476251 & -0.739014 \\ 1 & -0.134534 & -2.101568 & 1.814065 \\ 1 & -1.369433 & 0.061500 & 2.779083 \\ 1 & -3.108487 & 0.948686 & 0.908650 \\ 6 & 1.997935 & 1.186684 & 0.022680 \\ 8 & 2.866475 & 1.945539 & 0.109458 \\ 8 & -2.909470 & -0.596318 & -1.523082 \\ 1 & -3.526450 & 0.146332 & -1.497101 \\ 6 & -0.924634 & 2.898895 & 0.247462 \\ 8 & -0.565615 & 2.082445 & -0.578025 \\ 1 & -0.991167 & 2.652913 & 1.320071 \\ 1 & -1.198784 & 3.924986 & -0.055155 \\ 6 & 1.793660 & -1.486235 & -0.147153 \\ 8 & 2.544034 & -2.367026 & -0.165263\end{array}$

2b

$$
\begin{array}{ll}
\mathrm{E}(\mathrm{B} 3 \mathrm{LYP})=-704.426148875 & \mathrm{E}(\mathrm{CPCM})=-704.424155 \\
\mathrm{E}(\mathrm{MP} 2)=-702.07039090888 & \mathrm{E}(\mathrm{CCSD}(\mathrm{T}))=-702.18212833
\end{array}
$$

$\begin{array}{cccc}44 & 0.709357 & -0.008453 & -0.152334 \\ 1 & 1.030161 & -0.017279 & -1.693985 \\ 6 & -1.171463 & -1.351599 & -0.093001 \\ 6 & -0.942413 & -1.117331 & 1.326021 \\ 6 & -1.871007 & -0.165199 & 1.761368 \\ 6 & -2.744907 & 0.173733 & 0.683175 \\ 6 & -2.330937 & -0.562541 & -0.428110 \\ 1 & -0.954711 & -2.277960 & -0.612893 \\ 1 & -0.303249 & -1.708577 & 1.969776 \\ 1 & -1.941455 & 0.227752 & 2.770599 \\ 1 & -3.585308 & 0.859710 & 0.723528 \\ 6 & 2.260318 & 1.087720 & 0.025689 \\ 8 & 3.204108 & 1.748814 & 0.112611 \\ 8 & -2.873471 & -0.582772 & -1.669365 \\ 1 & -3.633759 & 0.012913 & -1.690674 \\ 6 & -1.073976 & 2.506236 & 0.250613 \\ 8 & -0.442240 & 1.830911 & -0.550917 \\ 1 & -1.059368 & 2.299885 & 1.327540 \\ 1 & -1.651192 & 3.373635 & -0.105480 \\ 6 & 1.717737 & -1.572699 & -0.043850 \\ 8 & 2.339982 & -2.545460 & -0.001971\end{array}$




$\begin{array}{cccc}44 & 0.758378 & 0.017373 & -0.241711 \\ 1 & 1.558589 & 0.174459 & -1.593995 \\ 6 & -0.876038 & -1.568905 & -0.633629 \\ 6 & -1.135213 & -1.259403 & 0.764892 \\ 6 & -2.277031 & -0.439444 & 0.789196 \\ 6 & -2.758294 & -0.232715 & -0.538575 \\ 6 & -1.911630 & -0.924496 & -1.395543 \\ 1 & -0.389173 & -2.477481 & -0.970545 \\ 1 & -0.706562 & -1.765619 & 1.622445 \\ 6 & 2.013066 & 1.264475 & 0.462780 \\ 8 & 2.788791 & 2.007016 & 0.890672 \\ 1 & -1.996585 & -0.965292 & -2.475374 \\ 6 & -1.367474 & 2.311962 & -0.382466 \\ 8 & -0.426324 & 1.751801 & -0.929880 \\ 1 & -1.720530 & 2.038103 & 0.619044 \\ 1 & -1.867733 & 3.149255 & -0.892956 \\ 6 & 1.873668 & -1.428302 & 0.127446 \\ 8 & 2.569182 & -2.328260 & 0.333820 \\ 1 & -3.622740 & 0.365374 & -0.801691 \\ 8 & -2.877241 & 0.136528 & 1.867199 \\ 1 & -2.425492 & -0.158903 & 2.669253\end{array}$

3b

$$
\begin{array}{ll}
\mathrm{E}(\mathrm{B} 3 \mathrm{LYP})=-704.419354946 & \mathrm{E}(\mathrm{CPCM})=-704.415005 \\
\mathrm{E}(\mathrm{MP} 2)=-702.06655642004 & \mathrm{E}(\mathrm{CCSD}(\mathrm{T}))=-702.17612384
\end{array}
$$

$\begin{array}{cccc}44 & -0.619026 & -0.003907 & -0.119772 \\ 1 & -0.974813 & -0.324413 & -1.650259 \\ 6 & 2.407349 & 0.294907 & -0.231545 \\ 6 & 1.307687 & 1.233156 & -0.335820 \\ 6 & 0.860807 & 1.438383 & 1.027604 \\ 6 & 1.594132 & 0.557120 & 1.855892 \\ 6 & 2.571763 & -0.117135 & 1.088203 \\ 1 & 1.253400 & 1.966015 & -1.132430 \\ 1 & 0.277157 & 2.277764 & 1.383472 \\ 1 & 1.441241 & 0.432247 & 2.921928 \\ 6 & -2.179729 & -0.990401 & 0.414743 \\ 8 & -3.113086 & -1.582050 & 0.743544 \\ 6 & 0.365895 & -2.044353 & -0.980724 \\ 8 & 0.477896 & -1.984218 & 0.265596 \\ 1 & -0.405024 & -2.671584 & -1.454564 \\ 1 & 1.148463 & -1.641246 & -1.640734 \\ 6 & -1.622595 & 1.539636 & -0.446633 \\ 8 & -2.246321 & 2.483153 & -0.673487 \\ 8 & 3.089134 & -0.126889 & -1.323397 \\ 1 & 3.771708 & -0.752118 & -1.042458 \\ 1 & 3.232158 & -0.902618 & 1.436650\end{array}$




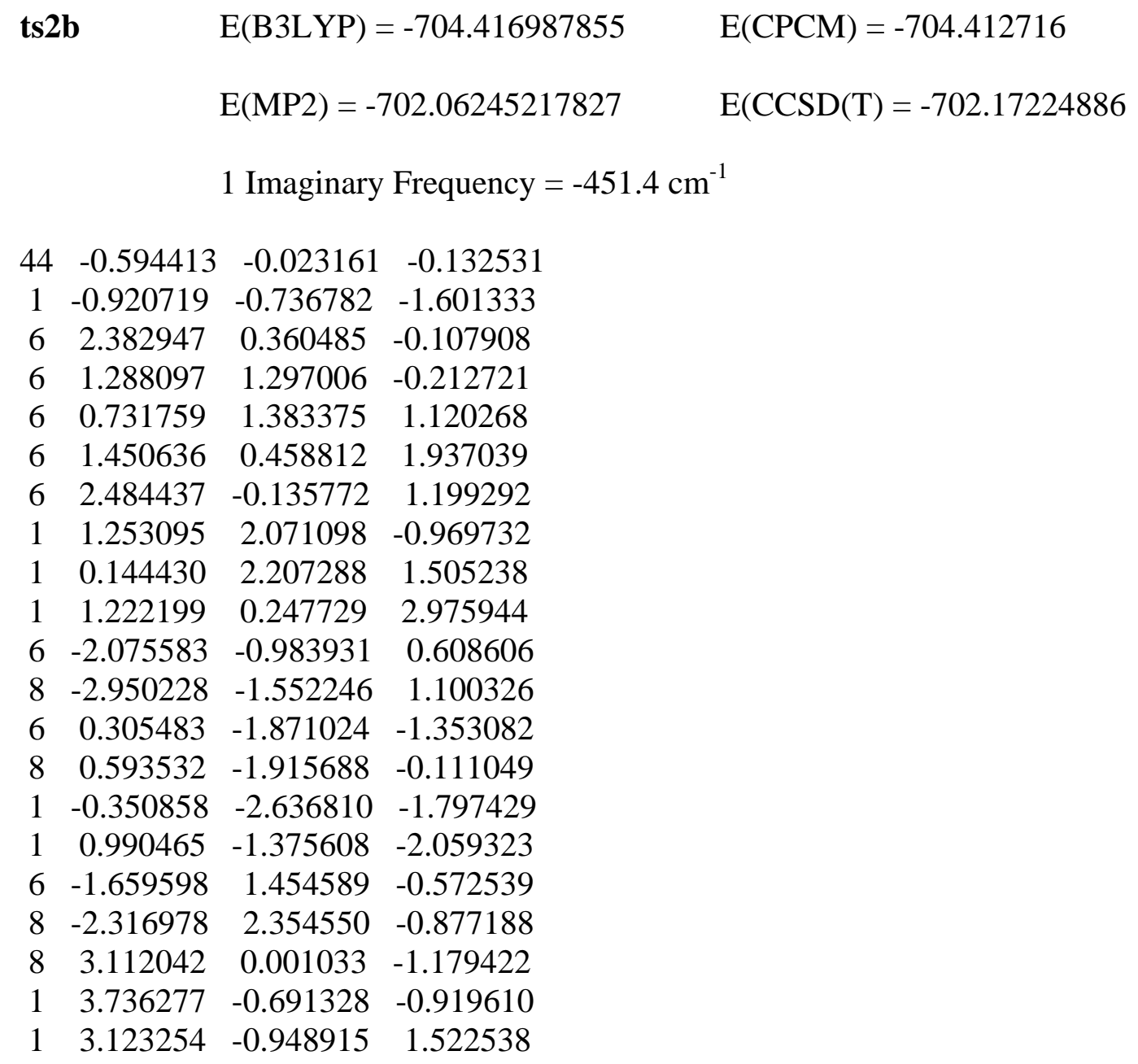

4b

$$
\begin{array}{ll}
\mathrm{E}(\mathrm{B} 3 \mathrm{LYP})=-704.426389748 & \mathrm{E}(\mathrm{CPCM})=-704.423083 \\
\mathrm{E}(\mathrm{MP} 2)=-702.07195325046 & \mathrm{E}(\mathrm{CCSD}(\mathrm{T}))=-702.18405313
\end{array}
$$

$\begin{array}{cccc}44 & -0.621683 & -0.034477 & -0.246317 \\ 1 & -0.947661 & -1.195554 & -1.929962 \\ 6 & 2.511746 & 0.464283 & 0.027614 \\ 6 & 1.404331 & 1.314711 & 0.062639 \\ 6 & 0.615712 & 0.923266 & 1.227532 \\ 6 & 1.376344 & -0.162025 & 1.882105 \\ 6 & 2.495272 & -0.440478 & 1.158445 \\ 1 & 1.304824 & 2.223807 & -0.514979 \\ 1 & 0.083234 & 1.651411 & 1.833364 \\ 1 & 1.034653 & -0.698246 & 2.759874 \\ 6 & -2.007272 & -0.770907 & 0.766832 \\ 8 & -2.816453 & -1.194676 & 1.476912 \\ 6 & -0.186876 & -2.042124 & -1.661737 \\ 8 & 0.475911 & -1.760410 & -0.495609 \\ 1 & -0.818693 & -2.947362 & -1.631812\end{array}$




$\begin{array}{rrrr}1 & 0.457631 & -2.065668 & -2.556761 \\ 6 & -1.605011 & 1.584346 & -0.508378 \\ 8 & -2.192257 & 2.555327 & -0.727866 \\ 8 & 3.449172 & 0.498329 & -0.926711 \\ 1 & 4.085725 & -0.215753 & -0.783421 \\ 1 & 3.197856 & -1.250636 & 1.317538\end{array}$
ts3b $\quad \mathrm{E}(\mathrm{B} 3 \mathrm{LYP})=-704.419723136 \quad \mathrm{E}(\mathrm{CPCM})=-704.414171$
$\mathrm{E}(\mathrm{MP} 2)=-702.07084957577 \quad \mathrm{E}(\mathrm{CCSD}(\mathrm{T})=-702.18211764$
1 Imaginary Frequency $=-145.0 \mathrm{~cm}^{-1}$

$\begin{array}{crcc}44 & 0.506965 & 0.089301 & -0.185327 \\ 1 & 0.654678 & 1.710761 & -1.549459 \\ 6 & -2.251622 & -0.584045 & -0.140397 \\ 6 & -1.197855 & -1.478380 & -0.430523 \\ 6 & -0.453671 & -1.619999 & 0.807248 \\ 6 & -1.229075 & -0.927858 & 1.850829 \\ 6 & -2.309721 & -0.320768 & 1.282918 \\ 1 & -1.061481 & -2.026371 & -1.353081 \\ 1 & 0.196629 & -2.456407 & 1.039294 \\ 1 & -0.920551 & -0.861636 & 2.887979 \\ 6 & 1.748081 & 0.817876 & 1.041412 \\ 8 & 2.447110 & 1.180959 & 1.885896 \\ 6 & -0.188105 & 2.367389 & -1.114306 \\ 8 & -0.905950 & 1.688314 & -0.146640 \\ 1 & 0.329367 & 3.269413 & -0.747850 \\ 1 & -0.778267 & 2.622180 & -2.010858 \\ 6 & 1.799400 & -1.110628 & -0.880734 \\ 8 & 2.582755 & -1.792244 & -1.388202 \\ 8 & -2.946674 & 0.115518 & -1.040036 \\ 1 & -2.646576 & 1.039267 & -0.840780 \\ 1 & -3.002768 & 0.371654 & 1.742324\end{array}$

ts1c $\quad \mathrm{E}(\mathrm{B} 3 \mathrm{LYP})=-704.414276147 \quad \mathrm{E}(\mathrm{CPCM})=-704.410229$

$$
\begin{array}{ll}
\mathrm{E}(\mathrm{MP} 2)=-702.06548546699 & \mathrm{E}(\mathrm{CCSD}(\mathrm{T}))=-702.1776559 \\
\mathrm{G}=-704.313471 & \mathrm{G}(\mathrm{CD})=-704.316134 \\
\mathrm{G}(\mathrm{OD})=-704.316931 & \mathrm{G}(\mathrm{CD}-\mathrm{OD})=-704.319595
\end{array}
$$

1 Imaginary Frequency $=-89.8 \mathrm{~cm}^{-1}$

$\begin{array}{cccc}44 & 0.457685 & 0.028612 & -0.138194 \\ 1 & 0.548302 & 1.166415 & -1.585990 \\ 6 & -2.252314 & -0.487823 & 0.077855\end{array}$




$\begin{array}{lrrr}6 & -1.339942 & -1.473782 & -0.511877 \\ 6 & -0.514375 & -1.936762 & 0.543655 \\ 6 & -0.768564 & -1.098286 & 1.676835 \\ 6 & -1.931147 & -0.302061 & 1.413266 \\ 1 & -1.473348 & -1.930429 & -1.484050 \\ 1 & 0.144904 & -2.794355 & 0.529487 \\ 1 & -0.269136 & -1.194997 & 2.633380 \\ 6 & 1.669867 & 1.101556 & 0.886526 \\ 8 & 2.349458 & 1.726028 & 1.572975 \\ 6 & -0.316474 & 2.021449 & -1.385084 \\ 8 & -0.916392 & 1.872026 & -0.196294 \\ 1 & 0.315746 & 2.918387 & -1.512896 \\ 1 & -0.947442 & 1.855181 & -2.277670 \\ 6 & 1.813684 & -1.116287 & -0.738991 \\ 8 & 2.649149 & -1.802906 & -1.145705 \\ 8 & -3.038596 & 0.304413 & -0.673603 \\ 1 & -2.647910 & 1.196218 & -0.502918 \\ 1 & -2.322633 & 0.480141 & 2.049131\end{array}$

ts1d

$$
\begin{array}{ll}
\mathrm{E}(\mathrm{B} 3 \mathrm{LYP})=-704.457124844 & \mathrm{E}(\mathrm{CPCM})=-704.453058 \\
\mathrm{E}(\mathrm{MP} 2)=-702.11412282014 & \mathrm{E}(\mathrm{CCSD}(\mathrm{T}))=-702.21058677 \\
\mathrm{G}=-704.357747 & \mathrm{G}(\mathrm{CD})=-704.359919 \\
\mathrm{G}(\mathrm{OD})=-704.360256 & \mathrm{G}(\mathrm{CD}-\mathrm{OD})=-704.362434
\end{array}
$$

1 Imaginary Frequency $=-746.8 \mathrm{~cm}^{-1}$

$\begin{array}{crcc}44 & 0.476068 & -0.060134 & 0.005180 \\ 1 & -0.212249 & 1.449596 & -0.398529 \\ 6 & -0.927495 & -1.656761 & -0.893163 \\ 6 & 0.057502 & -2.304531 & -0.073707 \\ 6 & -0.119877 & -1.853448 & 1.265959 \\ 6 & -1.211930 & -0.923349 & 1.287474 \\ 6 & -1.790175 & -0.877720 & -0.039091 \\ 1 & -1.089966 & -1.810587 & -1.951251 \\ 1 & 0.793264 & -3.024034 & -0.406962 \\ 1 & 0.462088 & -2.168617 & 2.122055 \\ 1 & -1.622805 & -0.431794 & 2.158828 \\ 6 & 1.562207 & 0.850982 & 1.277405 \\ 8 & 2.201330 & 1.377981 & 2.080511 \\ 8 & -2.819632 & -0.186197 & -0.439059 \\ 1 & -2.731312 & 0.880685 & -0.184407 \\ 6 & -1.272510 & 2.381562 & -0.642005 \\ 8 & -2.321958 & 2.121072 & 0.038749 \\ 1 & -0.650262 & 3.226030 & -0.315116 \\ 1 & -1.272792 & 2.213919 & -1.731734 \\ 6 & 1.780649 & 0.113455 & -1.373166\end{array}$


2c

$$
\begin{aligned}
& E(B 3 L Y P)=-704.475242258 \\
& E(M P 2)=-702.14297176758
\end{aligned}
$$

$\mathrm{E}(\mathrm{CCSD}(\mathrm{T})=-702.23594092$

a2 complete system $\mathrm{E}(\mathrm{B} 3 \mathrm{~L} Y \mathrm{P})=-1514.18288671 \quad \mathrm{E}(\mathrm{CPCM})=-1514.166744$

$$
\begin{array}{ll}
G=-1513.810169 & G(C D)=-1513.812550 \\
G(O D)=-1513.813750 & G(C D-O D)=-1513.816131
\end{array}
$$
$\begin{array}{llll}44 & -0.010407 & -0.701598 & 1.464969\end{array}$
$\begin{array}{llll}1 & 0.096776 & -2.181941 & 2.061784\end{array}$
$\begin{array}{llll}6 & -1.181510 & -0.858187 & -0.547802\end{array}$
$\begin{array}{llll}6 & -0.701000 & 0.521769 & -0.428948\end{array}$
$\begin{array}{llll}6 & 0.727448 & 0.495626 & -0.410404\end{array}$
$\begin{array}{lllll}6 & 1.161518 & -0.896931 & -0.534033\end{array}$
$\begin{array}{llll}6 & -0.025276 & -1.680848 & -0.698901\end{array}$
$\begin{array}{llll}6 & 1.292194 & -0.257996 & 2.759468\end{array}$
$\begin{array}{llll}8 & 2.110061 & 0.005978 & 3.533844\end{array}$
$8 \quad-0.096292-2.999401-0.981681$
$1 \quad 0.786570 \quad-3.385489-0.877204$
$\begin{array}{llll}6 & -1.411514 & -0.497093 & 2.715294\end{array}$
$\begin{array}{llll}8 & -2.288160 & -0.394183 & 3.462516\end{array}$
$\begin{array}{llll}6 & -2.569316 & -1.324027 & -0.798123\end{array}$ 


$\begin{array}{rrrr}6 & -3.092494 & -2.436254 & -0.119629 \\ 1 & -2.486107 & -2.930320 & 0.632519 \\ 6 & -4.376182 & -2.900932 & -0.400236 \\ 1 & -4.765328 & -3.760327 & 0.138284 \\ 6 & -5.161357 & -2.261845 & -1.362259 \\ 1 & -6.163924 & -2.621049 & -1.576311 \\ 6 & -4.649641 & -1.158572 & -2.046267 \\ 1 & -5.250236 & -0.656627 & -2.799552 \\ 6 & -3.363170 & -0.694885 & -1.770532 \\ 1 & -2.971323 & 0.158996 & -2.313074 \\ 6 & -1.554554 & 1.737337 & -0.491301 \\ 6 & -2.679142 & 1.905542 & 0.333156 \\ 1 & -2.919580 & 1.151300 & 1.074039 \\ 6 & -3.486108 & 3.035949 & 0.216684 \\ 1 & -4.347353 & 3.147683 & 0.869018 \\ 6 & -3.188596 & 4.020948 & -0.727511 \\ 1 & -3.816726 & 4.902784 & -0.814800 \\ 6 & -2.078511 & 3.863194 & -1.557831 \\ 1 & -1.838386 & 4.620107 & -2.299097 \\ 6 & -1.270939 & 2.731388 & -1.443488 \\ 1 & -0.412970 & 2.614175 & -2.097186 \\ 6 & 1.633028 & 1.674240 & -0.403013 \\ 6 & 2.600886 & 1.811248 & -1.412020 \\ 1 & 2.694512 & 1.042060 & -2.171981 \\ 6 & 3.437966 & 2.927134 & -1.450019 \\ 1 & 4.180383 & 3.014089 & -2.238239 \\ 6 & 3.322138 & 3.925188 & -0.481960 \\ 1 & 3.976041 & 4.792041 & -0.509332 \\ 6 & 2.361513 & 3.800676 & 0.524476 \\ 1 & 2.265587 & 4.570433 & 1.284771 \\ 6 & 1.526082 & 2.686113 & 0.564980 \\ 1 & 0.785568 & 2.590038 & 1.352506 \\ 6 & 2.532691 & -1.420528 & -0.762043 \\ 6 & 3.604092 & -1.145108 & 0.104126 \\ 1 & 3.434436 & -0.541953 & 0.988859 \\ 6 & 4.877137 & -1.646649 & -0.156491 \\ 1 & 5.690178 & -1.421892 & 0.527681 \\ 6 & 5.108929 & -2.443450 & -1.281157 \\ 1 & 6.101306 & -2.839300 & -1.475652 \\ 6 & 4.056875 & -2.725175 & -2.151347 \\ 1 & 4.224816 & -3.335494 & -3.034113 \\ 6 & 2.782078 & -2.212681 & -1.898986 \\ 1 & 1.977224 & -2.409322 & -2.602297\end{array}$

$1 \mathbf{b 2} \mathrm{E}(\mathrm{B} 3 \mathrm{LYP})=-1628.697252090 \quad \mathrm{E}(\mathrm{CPCM})=-1628.677687$

$\begin{array}{cccc}44 & 0.014081 & -0.555361 & 1.406121 \\ 1 & 0.494937 & -1.994022 & 1.929455 \\ 6 & -1.129160 & -0.877193 & -0.600812\end{array}$ 


$\begin{array}{rrrr}6 & -0.976269 & 0.566444 & -0.408352 \\ 6 & 0.421133 & 0.865147 & -0.401281 \\ 6 & 1.160280 & -0.381862 & -0.602272 \\ 6 & 0.181486 & -1.414773 & -0.806616 \\ 6 & 1.174459 & 0.158286 & 2.715297 \\ 8 & 1.908078 & 0.591811 & 3.499156 \\ 8 & 0.386505 & -2.687762 & -1.170840 \\ 1 & 1.270909 & -3.010561 & -0.887635 \\ 6 & 3.159774 & -3.543425 & 0.892405 \\ 8 & 2.686717 & -3.878770 & -0.175279 \\ 1 & 4.006782 & -4.099480 & 1.335817 \\ 1 & 2.770444 & -2.683386 & 1.462447 \\ 6 & -1.382011 & -0.768579 & 2.664913 \\ 8 & -2.246944 & -0.923985 & 3.416659 \\ 6 & -2.381636 & -1.631118 & -0.862791 \\ 6 & -2.618605 & -2.875988 & -0.257926 \\ 1 & -1.892198 & -3.269987 & 0.444960 \\ 6 & -3.772109 & -3.600797 & -0.550995 \\ 1 & -3.939713 & -4.560116 & -0.069600 \\ 6 & -4.710751 & -3.096350 & -1.453898 \\ 1 & -5.611891 & -3.660108 & -1.677926 \\ 6 & -4.482505 & -1.863848 & -2.066404 \\ 1 & -5.202943 & -1.464025 & -2.774418 \\ 6 & -3.326614 & -1.138251 & -1.776868 \\ 1 & -3.154866 & -0.184512 & -2.264301 \\ 6 & -2.082037 & 1.560726 & -0.404565 \\ 6 & -3.182777 & 1.455473 & 0.460923 \\ 1 & -3.217927 & 0.651397 & 1.187489 \\ 6 & -4.226704 & 2.377259 & 0.402073 \\ 1 & -5.065888 & 2.279258 & 1.084716 \\ 6 & -4.193865 & 3.422296 & -0.523668 \\ 1 & -5.006930 & 4.141287 & -0.566142 \\ 6 & -3.108367 & 3.535871 & -1.392891 \\ 1 & -3.071896 & 4.342593 & -2.119483 \\ 6 & -2.063924 & 2.612801 & -1.336171 \\ 1 & -1.226145 & 2.705255 & -2.019642 \\ 6 & 1.027736 & 2.221470 & -0.343345 \\ 6 & 1.907958 & 2.630344 & -1.359069 \\ 1 & 2.159052 & 1.941459 & -2.159008 \\ 6 & 2.457707 & 3.912801 & -1.351881 \\ 1 & 3.136956 & 4.209656 & -2.145943 \\ 6 & 2.137281 & 4.808532 & -0.331221 \\ 1 & 2.568088 & 5.805627 & -0.323430 \\ 6 & 1.260828 & 4.413702 & 0.682231 \\ 1 & 1.007178 & 5.102676 & 1.482720 \\ 6 & 0.711521 & 3.132903 & 0.677019 \\ 1 & 0.035116 & 2.827300 & 1.468714 \\ 6 & 2.610643 & -0.534394 & -0.884372 \\ 6 & 3.605751 & -0.068212 & -0.009587 \\ 1 & 3.318144 & 0.409421 & 0.920813\end{array}$




$\begin{array}{rrrc}6 & 4.956924 & -0.215687 & -0.322929 \\ 1 & 5.709992 & 0.153979 & 0.367325 \\ 6 & 5.341842 & -0.835978 & -1.514233 \\ 1 & 6.394571 & -0.953595 & -1.754253 \\ 6 & 4.363552 & -1.303244 & -2.392459 \\ 1 & 4.649807 & -1.786815 & -3.321990 \\ 6 & 3.011404 & -1.151074 & -2.082904 \\ 1 & 2.256327 & -1.517444 & -2.771669\end{array}$

ts1d2

$$
\begin{array}{ll}
E(B 3 L Y P)=-1628.684155030 & E(C P C M)=-1628.664806 \\
G=-1628.287932 & G(C D)=-1628.290072 \\
G(O D)=-1628.290552 & G(C D-O D)=-1628.292703
\end{array}
$$

1 Imaginary Frequency $=-611.2 \mathrm{~cm}^{-1}$

$\begin{array}{cccc}44 & 0.022074 & -0.664495 & 1.288253 \\ 1 & 0.377134 & -2.282673 & 1.675390 \\ 6 & -1.184221 & -0.670162 & -0.687686 \\ 6 & -0.798492 & 0.701068 & -0.390536 \\ 6 & 0.643113 & 0.772984 & -0.405939 \\ 6 & 1.168289 & -0.545043 & -0.708993 \\ 6 & 0.026442 & -1.407616 & -0.966366 \\ 6 & 1.302534 & -0.154845 & 2.603634 \\ 8 & 2.095890 & 0.172995 & 3.376157 \\ 8 & 0.054498 & -2.664595 & -1.335683 \\ 1 & 0.619860 & -3.297136 & -0.663404 \\ 6 & 0.647180 & -3.688239 & 1.489362 \\ 8 & 1.220541 & -3.922438 & 0.376705 \\ 1 & 1.257152 & -3.727561 & 2.403684 \\ 1 & -0.406619 & -3.981349 & 1.640167 \\ 6 & -1.388226 & -0.678109 & 2.568171 \\ 8 & -2.267078 & -0.692747 & 3.318065 \\ 6 & -2.545742 & -1.190080 & -0.970054 \\ 6 & -2.967199 & -2.429741 & -0.464136 \\ 1 & -2.299589 & -2.995097 & 0.176477 \\ 6 & -4.226505 & -2.936993 & -0.777687 \\ 1 & -4.534872 & -3.896628 & -0.372836 \\ 6 & -5.088331 & -2.215993 & -1.606951 \\ 1 & -6.071046 & -2.610255 & -1.849130 \\ 6 & -4.676727 & -0.987664 & -2.124815 \\ 1 & -5.335744 & -0.421745 & -2.777064 \\ 6 & -3.415754 & -0.479399 & -1.812588 \\ 1 & -3.103288 & 0.472303 & -2.228573 \\ 6 & -1.718605 & 1.867293 & -0.315202 \\ 6 & -2.807028 & 1.907588 & 0.569993 \\ 1 & -2.965465 & 1.088509 & 1.261726 \\ 6 & -3.682706 & 2.992064 & 0.573021\end{array}$




$\begin{array}{rrrr}1 & -4.515559 & 3.005320 & 1.269980 \\ 6 & -3.490536 & 4.055436 & -0.311363 \\ 1 & -4.172934 & 4.900412 & -0.306641 \\ 6 & -2.415468 & 4.025242 & -1.200366 \\ 1 & -2.257019 & 4.845359 & -1.894668 \\ 6 & -1.537046 & 2.941682 & -1.202800 \\ 1 & -0.705375 & 2.925881 & -1.899450 \\ 6 & 1.440594 & 2.022819 & -0.295351 \\ 6 & 2.349410 & 2.347081 & -1.316164 \\ 1 & 2.487796 & 1.663272 & -2.147220 \\ 6 & 3.073534 & 3.538419 & -1.269943 \\ 1 & 3.772018 & 3.772530 & -2.068061 \\ 6 & 2.904280 & 4.422355 & -0.203382 \\ 1 & 3.472392 & 5.347239 & -0.164788 \\ 6 & 2.002086 & 4.109436 & 0.815848 \\ 1 & 1.865044 & 4.789941 & 1.651134 \\ 6 & 1.275108 & 2.921167 & 0.770189 \\ 1 & 0.574637 & 2.681373 & 1.563458 \\ 6 & 2.578110 & -0.932710 & -0.962953 \\ 6 & 3.633601 & -0.513194 & -0.135964 \\ 1 & 3.424008 & 0.085486 & 0.743604 \\ 6 & 4.949363 & -0.865758 & -0.426948 \\ 1 & 5.749671 & -0.534394 & 0.228469 \\ 6 & 5.238462 & -1.646883 & -1.548392 \\ 1 & 6.264522 & -1.925252 & -1.770863 \\ 6 & 4.199020 & -2.072614 & -2.375208 \\ 1 & 4.410652 & -2.684558 & -3.247340 \\ 6 & 2.880413 & -1.720183 & -2.087259 \\ 1 & 2.076850 & -2.065382 & -2.728145\end{array}$

$2 c 2$

$\mathrm{E}(\mathrm{B} 3 \mathrm{LYP})=-1628.70459439$

$\mathrm{E}(\mathrm{CPCM})=-1628.686868$

$\begin{array}{cccc}44 & 0.021845 & -0.630467 & 1.262775 \\ 1 & 0.394512 & -2.577856 & 1.680603 \\ 6 & -1.191956 & -0.701954 & -0.683486 \\ 6 & -0.821396 & 0.654202 & -0.383249 \\ 6 & 0.639332 & 0.753440 & -0.421153 \\ 6 & 1.179863 & -0.534131 & -0.733839 \\ 6 & 0.040044 & -1.435895 & -1.070306 \\ 6 & 1.350214 & -0.111791 & 2.547136 \\ 8 & 2.156266 & 0.268778 & 3.280415 \\ 8 & 0.104649 & -2.620013 & -1.441887 \\ 1 & 0.993264 & -3.712824 & -0.316081 \\ 6 & 0.533361 & -3.708884 & 1.560543 \\ 8 & 1.381073 & -4.042959 & 0.522549 \\ 1 & 0.980261 & -4.025654 & 2.506856 \\ 1 & -0.482628 & -4.122399 & 1.473633 \\ 6 & -1.367129 & -0.543746 & 2.577524 \\ 8 & -2.229379 & -0.467005 & 3.342185\end{array}$




$\begin{array}{rrrr}6 & -2.543895 & -1.249663 & -0.948545 \\ 6 & -2.863497 & -2.561702 & -0.559948 \\ 1 & -2.121430 & -3.155710 & -0.039783 \\ 6 & -4.113012 & -3.107936 & -0.845306 \\ 1 & -4.339165 & -4.122924 & -0.531208 \\ 6 & -5.068229 & -2.357514 & -1.533798 \\ 1 & -6.042908 & -2.782587 & -1.755517 \\ 6 & -4.757190 & -1.060120 & -1.942289 \\ 1 & -5.487047 & -0.470998 & -2.490414 \\ 6 & -3.506945 & -0.511514 & -1.657193 \\ 1 & -3.276163 & 0.492147 & -1.995420 \\ 6 & -1.732924 & 1.829151 & -0.312117 \\ 6 & -2.785179 & 1.919500 & 0.611187 \\ 1 & -2.928129 & 1.134789 & 1.343490 \\ 6 & -3.651132 & 3.011983 & 0.597065 \\ 1 & -4.455616 & 3.065442 & 1.324641 \\ 6 & -3.487987 & 4.029343 & -0.344987 \\ 1 & -4.164197 & 4.879174 & -0.354374 \\ 6 & -2.449525 & 3.947427 & -1.273475 \\ 1 & -2.313075 & 4.731805 & -2.012179 \\ 6 & -1.577309 & 2.859300 & -1.255722 \\ 1 & -0.771963 & 2.805757 & -1.980891 \\ 6 & 1.392788 & 2.030862 & -0.325833 \\ 6 & 2.277510 & 2.377498 & -1.360494 \\ 1 & 2.432146 & 1.690119 & -2.185760 \\ 6 & 2.956673 & 3.595180 & -1.333657 \\ 1 & 3.636073 & 3.848145 & -2.142337 \\ 6 & 2.768841 & 4.480777 & -0.271256 \\ 1 & 3.303527 & 5.425827 & -0.247186 \\ 6 & 1.891648 & 4.144217 & 0.762025 \\ 1 & 1.740689 & 4.826351 & 1.593487 \\ 6 & 1.205474 & 2.931350 & 0.733914 \\ 1 & 0.522743 & 2.673303 & 1.536554 \\ 6 & 2.598252 & -0.904389 & -0.947778 \\ 6 & 3.634718 & -0.395295 & -0.144077 \\ 1 & 3.402243 & 0.268912 & 0.680519 \\ 6 & 4.961453 & -0.738803 & -0.388746 \\ 1 & 5.744349 & -0.337659 & 0.248444 \\ 6 & 5.284421 & -1.598839 & -1.441432 \\ 1 & 6.319679 & -1.869097 & -1.628608 \\ 6 & 4.266588 & -2.112619 & -2.244381 \\ 1 & 4.504774 & -2.785612 & -3.063081 \\ 6 & 2.935539 & -1.772769 & -2.001871 \\ 1 & 2.149498 & -2.188348 & -2.620322\end{array}$

b complete system $\quad \mathrm{E}(\mathrm{B} 3 \mathrm{LYP})=-1512.96699502 \quad \mathrm{E}(\mathrm{CPCM})=-1512.954661$

$44-0.002443-0.748732 \quad 1.361751$

$\begin{array}{llll}6 & -1.212956 & -0.877583 & -0.573726\end{array}$ 


$\begin{array}{rrrr}6 & -0.733423 & 0.454302 & -0.406341 \\ 6 & 0.740388 & 0.429724 & -0.430476 \\ 6 & 1.177593 & -0.911168 & -0.617567 \\ 6 & -0.036554 & -1.759168 & -0.859374 \\ 6 & 1.379062 & -0.369371 & 2.634024 \\ 8 & 2.229970 & -0.064316 & 3.353036 \\ 8 & -0.066840 & -2.970139 & -1.071341 \\ 6 & -1.373048 & -0.487240 & 2.666373 \\ 8 & -2.228354 & -0.286330 & 3.417107 \\ 6 & -2.600005 & -1.354358 & -0.770192 \\ 6 & -2.990004 & -2.603772 & -0.255822 \\ 1 & -2.271697 & -3.197683 & 0.297621 \\ 6 & -4.280189 & -3.087386 & -0.463762 \\ 1 & -4.563371 & -4.052356 & -0.053274 \\ 6 & -5.202444 & -2.340396 & -1.199324 \\ 1 & -6.207795 & -2.718414 & -1.361569 \\ 6 & -4.820302 & -1.108589 & -1.732959 \\ 1 & -5.525299 & -0.525552 & -2.318830 \\ 6 & -3.531263 & -0.619867 & -1.524112 \\ 1 & -3.244617 & 0.332284 & -1.955887 \\ 6 & -1.540410 & 1.703931 & -0.433323 \\ 6 & -2.582644 & 1.948801 & 0.474021 \\ 1 & -2.794487 & 1.233014 & 1.258767 \\ 6 & -3.349312 & 3.108843 & 0.377555 \\ 1 & -4.146819 & 3.283289 & 1.093748 \\ 6 & -3.096578 & 4.039873 & -0.631900 \\ 1 & -3.695975 & 4.942591 & -0.705351 \\ 6 & -2.068153 & 3.803550 & -1.544853 \\ 1 & -1.862811 & 4.519589 & -2.335184 \\ 6 & -1.293350 & 2.648152 & -1.444660 \\ 1 & -0.493585 & 2.474553 & -2.156949 \\ 6 & 1.588055 & 1.649669 & -0.431861 \\ 6 & 2.490359 & 1.853075 & -1.489226 \\ 1 & 2.583478 & 1.100219 & -2.265495 \\ 6 & 3.266020 & 3.010528 & -1.545481 \\ 1 & 3.957952 & 3.153169 & -2.370354 \\ 6 & 3.157895 & 3.977327 & -0.544127 \\ 1 & 3.767455 & 4.875331 & -0.584594 \\ 6 & 2.263679 & 3.783242 & 0.510696 \\ 1 & 2.174799 & 4.529551 & 1.294633 \\ 6 & 1.480698 & 2.631210 & 0.565277 \\ 1 & 0.786589 & 2.482464 & 1.385758 \\ 6 & 2.559327 & -1.427034 & -0.739856 \\ 6 & 3.631773 & -0.878109 & -0.010865 \\ 1 & 3.453509 & -0.064558 & 0.682613 \\ 6 & 4.924390 & -1.372563 & -0.160892 \\ 1 & 5.734330 & -0.935888 & 0.416329 \\ 6 & 5.178254 & -2.427230 & -1.041497 \\ 1 & 6.187033 & -2.813569 & -1.155673 \\ 6 & 4.124496 & -2.983896 & -1.766227\end{array}$


$1 \quad 4.308349 \quad-3.807458 \quad-2.450298$

$\begin{array}{llll}6 & 2.827082 & -2.493718 & -1.618543\end{array}$

$\begin{array}{llll}1 & 2.011615 & -2.944775 & -2.169345\end{array}$

\subsection{Jaguar Calculations}

\section{a2 complete system}

$\mathrm{Ru} \quad-0.0088078237$

$\mathrm{H} \quad 0.1016016600$

C $\quad-1.1846693228$

C $\quad-0.7018593469$

C $\quad 0.7261061406$

C $\quad 1.1569971274$

C $\quad-0.0312256628$

C $\quad 1.3029406501$

O 2.1327926831

O $\quad-0.1018429357$

$\mathrm{H} \quad 0.7821074695$

C $\quad-1.4067112248$

O $\quad-2.2826845386$

C $\quad-2.5724810400$

C $\quad-3.1154890649$

$\mathrm{H} \quad-2.5278458321$

C $\quad-4.3957056804$

$\mathrm{H} \quad-4.8013337554$

C $\quad-5.1559104179$

$\mathrm{H} \quad-6.1555573291$

C $\quad-4.6239966825$

$\mathrm{H} \quad-5.2056767669$

C $\quad-3.3417396252$

$\mathrm{H} \quad-2.9326766904$

C $\quad-1.5574369010$

C $\quad-2.6817303242$

$\mathrm{H} \quad-2.9199122565$

C $\quad-3.4925438283$

$\mathrm{H} \quad-4.3554492176$

C $\quad-3.1981586894$

$\mathrm{H} \quad-3.8305469045$

C $\quad-2.0871090281$

$\mathrm{H} \quad-1.8492195586$

C $\quad-1.2757094736$

$\mathrm{H} \quad-0.4173904353$

C $\quad 1.6337679701$

C $\quad 2.5831180761$

$\mathrm{H} \quad 2.6628314165$

C $\quad 3.4182043675$

$$
\mathrm{E}(\mathrm{B} 3 \mathrm{LYP})=-1514.19480418672
$$

$-0.7081836751$

1.4668108157

$-2.1986620816$

$-0.8562556583$

0.5221165605

0.4941146106

$-0.8983679025$

$-1.6821295769$

$-0.2733211020$

$-0.0165849860$

$-3.0024160483$

$-3.3860133966$

$-0.5076759500$

$-0.4085652743$

$-1.3132784032$

$-2.4139097363$

$-2.9040175581$

$-2.8733277815$

$-3.7251082686$

$-2.2406977663$

$-2.5976698466$

$-1.1481486056$

$-0.6513076350$

$-0.6887977426$

0.1568986280

1.7363893142

1.9029183357

1.1485633711

3.0295929165

3.1400656968

4.0123582454

4.8911845480

3.8570821296

4.6128097727

2.7292395722

2.6145470435

1.6711049224

1.8025239653

1.0297805264

2.9180747014
2.0530510086

$-0.5379068886$

$-0.4078064694$

$-0.3921490984$

$-0.5226605092$

$-0.6814589373$

2.7571765358

3.5222448474

$-0.9603610583$

$-0.8553070381$

2.7268503391

3.4764106060

$-0.8015741504$

$-0.1220534963$

0.6474058527

$-0.4241778657$

0.1147767144

$-1.4092621631$

$-1.6415602929$

$-2.0943676565$

$-2.8660359033$

$-1.7960565738$

$-2.3396499071$

$-0.4701600844$

0.3537452952

1.0957643155

0.2332423895

0.8840535801

$-0.7135065732$

$-0.8049393193$

$-1.5420494924$

$-2.2857167629$

$-1.4234952037$

$-2.0772143689$

$-0.3990526903$

$-1.4259190691$

$-2.1843519976$

$-1.4854118169$ 


$\begin{array}{lrrr}\mathrm{H} & 4.1461397646 & 3.0017779480 & -2.2878471664 \\ \mathrm{C} & 3.3190891787 & 3.9206847199 & -0.5207880727 \\ \mathrm{H} & 3.9712555775 & 4.7886474082 & -0.5653111267 \\ \mathrm{C} & 2.3780394918 & 3.8007935141 & 0.5037856068 \\ \mathrm{H} & 2.2952914250 & 4.5753403861 & 1.2611752544 \\ \mathrm{C} & 1.5441035235 & 2.6863109900 & 0.5659394928 \\ \mathrm{H} & 0.8175836150 & 2.5948901670 & 1.3673739427 \\ \mathrm{C} & 2.5292505470 & -1.4178015932 & -0.7519134916 \\ \mathrm{C} & 3.6049786128 & -1.1200154434 & 0.1008319807 \\ \mathrm{H} & 3.4385817821 & -0.5054191432 & 0.9786089487 \\ \mathrm{C} & 4.8805302003 & -1.6104800752 & -0.1659146608 \\ \mathrm{H} & 5.6982029412 & -1.3669033354 & 0.5066832691 \\ \mathrm{C} & 5.1102311481 & -2.4180464329 & -1.2827988509 \\ \mathrm{H} & 6.1058146227 & -2.8040389063 & -1.4828300039 \\ \mathrm{C} & 4.0535932334 & -2.7231703349 & -2.1385393153 \\ \mathrm{H} & 4.2207951603 & -3.3427425563 & -3.0153637548 \\ \mathrm{C} & 2.7755216499 & -2.2232983121 & -1.8796195164 \\ \mathrm{H} & 1.9669867335 & -2.4359659879 & -2.5746034136\end{array}$
$\mathrm{H}_{2} \mathrm{C}=\mathrm{O}$
$E(B 3 L Y P)=-114.5031945005$
C $\quad 0.0000000000$
0.0000000000
0.6032571356
O $\quad 0.0000000000$
0.0000000000
$-0.6035329039$
$\mathrm{H} \quad 0.0000000000$
0.9378011551
1.1978128970
$\mathrm{H} \quad 0.0000000000$
$-0.9378011551$
1.1978128970

\section{$\mathrm{CH}_{3} \mathrm{OH}$}

$\mathrm{E}(\mathrm{B} 3 \mathrm{LYP})=-115.72395898281$

$\begin{array}{cc}\mathrm{H} & 1.0387920000 \\ \mathrm{H} & -1.1349790000 \\ \mathrm{C} & 0.6612730000 \\ \mathrm{O} & -0.7489590000 \\ \mathrm{H} & 1.0821590000 \\ \mathrm{H} & 1.0380590000\end{array}$

$-0.5409120000$

0.8928900000

$-0.7623540000$

0.0010540000

$-0.0194990000$

$-0.0000960000$

0.1219870000

$-0.0001050000$

0.9895020000

$-0.0017480000$

$-0.5451380000$

$-0.8907830000$

1b complete system

$\begin{array}{lc}\mathrm{Ru} & 0.0162798626 \\ \mathrm{H} & 0.4909747926 \\ \mathrm{C} & -1.1312658702 \\ \mathrm{C} & -0.9747670532 \\ \mathrm{C} & 0.4230375396 \\ \mathrm{C} & 1.1583646313 \\ \mathrm{C} & 0.1777552797 \\ \mathrm{C} & 1.1883433686 \\ \mathrm{O} & 1.9335937154 \\ \mathrm{O} & 0.3860534112\end{array}$

$\mathrm{E}(\mathrm{B} 3 \mathrm{LYP})=-1628.70901053075$
$-0.5625635391$
1.4055195498
$-2.0150426243$
1.9128100215
$-0.8714940362$
$-0.5945583009$
0.5695358540
$-0.3878096134$
0.8659563712
$-0.3841142730$
$-0.3807206428$
$-0.5962637581$
$-0.7912865810$
$-1.4139234328$
2.7147221361
3.4930913856
0.5624699765
$-2.6899018863$ 


\begin{tabular}{|c|c|c|c|}
\hline $\mathrm{H}$ & 1.2666703875 & -3.0064500287 & -0.8484211551 \\
\hline $\mathrm{C}$ & 3.2048438399 & -3.5039947750 & 0.9358352916 \\
\hline $\mathrm{O}$ & 2.6923449455 & -3.8513160314 & -0.1093786297 \\
\hline $\mathrm{H}$ & 4.0625454115 & -4.0609373662 & 1.3579062824 \\
\hline $\mathrm{H}$ & 2.8429401228 & -2.6318286815 & 1.5057340979 \\
\hline $\mathrm{C}$ & -1.3797106190 & -0.7785658551 & 2.6678727442 \\
\hline $\mathrm{O}$ & -2.2475947847 & -0.9388102846 & 3.4162278428 \\
\hline $\mathrm{C}$ & -2.3878432443 & -1.6132774033 & -0.8699400139 \\
\hline $\mathrm{C}$ & -2.6352558866 & -2.8641745655 & -0.2834414194 \\
\hline $\mathrm{H}$ & -1.9158930777 & -3.2715087236 & 0.4195876264 \\
\hline $\mathrm{C}$ & -3.7897214857 & -3.5786527858 & -0.5956133805 \\
\hline $\mathrm{H}$ & -3.9661031220 & -4.5442667060 & -0.1295078873 \\
\hline $\mathrm{C}$ & -4.7176315508 & -3.0573103363 & -1.4991786040 \\
\hline $\mathrm{H}$ & -5.6188622110 & -3.6146246981 & -1.7399537969 \\
\hline $\mathrm{C}$ & -4.4791199427 & -1.8181009671 & -2.0928113009 \\
\hline $\mathrm{H}$ & -5.1922202839 & -1.4062956812 & -2.8017795186 \\
\hline $\mathrm{C}$ & -3.3234516604 & -1.1011046434 & -1.7838497879 \\
\hline $\mathrm{H}$ & -3.1424899727 & -0.1426699323 & -2.2591068568 \\
\hline $\mathrm{C}$ & -2.0852658192 & 1.558645 & -0.383392 \\
\hline $\mathrm{C}$ & -3.1953377601 & 1.4345134395 & 0.4668636689 \\
\hline $\mathrm{H}$ & -3.2334068788 & 0.6227731662 & 1.1852372112 \\
\hline $\mathrm{C}$ & -4.2492240394 & 2.3434067449 & 0.3995995 \\
\hline $\mathrm{H}$ & -5.0979033231 & 2.2293986029 & 1.0683253163 \\
\hline $\mathrm{C}$ & -4.2164038973 & 3.3944468966 & -0.5183970497 \\
\hline $\mathrm{H}$ & -5.0390306162 & 4.1024246482 & -0.5691624917 \\
\hline $\mathrm{C}$ & -3.1210096280 & 3.5279344413 & -1.3712793934 \\
\hline $\mathrm{H}$ & -3.0851006817 & 4.3394523391 & -2.0929841028 \\
\hline $\mathrm{C}$ & -2.0664184280 & 2.6178606837 & -1.3061089391 \\
\hline $\mathrm{H}$ & -1.2220628490 & 2.7248698311 & -1.9791310035 \\
\hline $\mathrm{C}$ & 1.0326195624 & 2.2212589919 & -0.3377367920 \\
\hline $\mathrm{C}$ & 1.9048334729 & 2.6204001265 & 72151 \\
\hline $\mathrm{H}$ & 2.1500369259 & 1.9251005455 & -2.1596403786 \\
\hline $\mathrm{C}$ & 2.4546470997 & 3.9020312329 & -1.3732468234 \\
\hline $\mathrm{H}$ & 3.1274255608 & 4.1908579622 & -2.1762137560 \\
\hline $\mathrm{C}$ & 2.1426190040 & 4.8071896623 & -0.3591580475 \\
\hline $\mathrm{H}$ & 2.5730362182 & 5.8048325125 & -0.3649694031 \\
\hline $\mathrm{C}$ & 1.2746762996 & 4.4218453654 & 0.6645438002 \\
\hline $\mathrm{H}$ & 1.0267691773 & 5.1188107823 & 1.4602146456 \\
\hline $\mathrm{C}$ & 0.7255321920 & 3.1412307718 & 0.6766448160 \\
\hline $\mathrm{H}$ & 0.0549967070 & 2.8447306043 & 1.4771827873 \\
\hline $\mathrm{C}$ & 2.6078782136 & -0.5358507210 & -0.8849350200 \\
\hline $\mathrm{C}$ & 3.6089453368 & -0.0448801424 & -0.0312358949 \\
\hline $\mathrm{H}$ & 3.3295067802 & 0.4558162825 & 0.8899587519 \\
\hline C & 4.9575474849 & -0.1950411343 & -0.3540277746 \\
\hline $\mathrm{H}$ & 5.7154901014 & 0.1941613966 & 0.3204146097 \\
\hline $\mathrm{C}$ & 5.3348757447 & -0.8424082503 & -1.5331193126 \\
\hline $\mathrm{H}$ & 6.3859879989 & -0.9621230642 & -1.7801137273 \\
\hline $\mathrm{C}$ & 4.3500166353 & -1.3335908380 & -2.3902218230 \\
\hline $\mathrm{H}$ & 4.6290861386 & -1.8386592471 & -3.3108390695 \\
\hline $\mathrm{C}$ & 3.0004990718 & -1.1797024723 & -2.0715817370 \\
\hline
\end{tabular}




\section{2b complete system}

\begin{tabular}{|c|c|}
\hline $\mathrm{Ru}$ & -1.1159472721 \\
\hline $\mathrm{H}$ & -1.8276702702 \\
\hline $\mathrm{C}$ & 1.0029064603 \\
\hline C & -1.1497228643 \\
\hline $\mathrm{O}$ & -1.1752056825 \\
\hline $\mathrm{O}$ & 0.2126601103 \\
\hline $\mathrm{H}$ & 1.0460117836 \\
\hline $\mathrm{C}$ & 1.5968786184 \\
\hline $\mathrm{O}$ & 0.7508101669 \\
\hline $\mathrm{H}$ & 1.6128864579 \\
\hline $\mathrm{H}$ & 2.3791571858 \\
\hline $\mathrm{C}$ & -2.8583773052 \\
\hline $\mathrm{O}$ & -3.9665072929 \\
\hline $\mathrm{H}$ & -5.3147370263 \\
\hline $\mathrm{C}$ & -4.4318749450 \\
\hline $\mathrm{C}$ & -3.8330998108 \\
\hline $\mathrm{C}$ & -3.8802870883 \\
\hline $\mathrm{C}$ & -2.7074232025 \\
\hline $\mathrm{H}$ & -4.2489603515 \\
\hline $\mathrm{H}$ & -4.3298569575 \\
\hline $\mathrm{C}$ & -2.7430590472 \\
\hline $\mathrm{C}$ & -2.1362691701 \\
\hline $\mathrm{H}$ & -2.2611919617 \\
\hline $\mathrm{H}$ & -2.3280039883 \\
\hline $\mathrm{C}$ & -0.8933631228 \\
\hline $\mathrm{C}$ & 0.2816843593 \\
\hline C & -0.3921184424 \\
\hline $\mathrm{C}$ & 1.4357076289 \\
\hline $\mathrm{C}$ & -0.9796762895 \\
\hline $\mathrm{C}$ & 2.7741844755 \\
\hline $\mathrm{C}$ & 1.8919295907 \\
\hline $\mathrm{C}$ & -1.8007768477 \\
\hline $\mathrm{C}$ & -0.6239501911 \\
\hline $\mathrm{C}$ & 3.6532850640 \\
\hline $\mathrm{C}$ & 3.2285420544 \\
\hline $\mathrm{C}$ & 3.0310274173 \\
\hline $\mathrm{C}$ & 1.6165373866 \\
\hline $\mathrm{H}$ & -2.0573106914 \\
\hline $\mathrm{C}$ & -2.2788168125 \\
\hline $\mathrm{C}$ & -1.0955335821 \\
\hline $\mathrm{H}$ & 0.0292388748 \\
\hline $\mathrm{H}$ & 3.3587954162 \\
\hline $\mathrm{C}$ & 4.9073504335 \\
\hline $\mathrm{C}$ & 4.4794479569 \\
\hline $\mathrm{H}$ & 2.6171107968 \\
\hline
\end{tabular}

$\mathrm{E}(\mathrm{B}$ 3LYP $)=-1628.65862919074$

-0.5162422616
-1.8608245034
0.4751937989
0.0007746810
0.3208247017

1.4780387087

1.9511432641

$-0.2887448922$

3.3051851862

4.4190964580

$-0.9677411561$

$-0.6647280421$

2.9196730216

2.0507749880

3.6048877351

3.0446580592

1.2476561926

1.1621944801

$-3.4679592982$

$-2.9384703538$

$-1.9607214437$

$-3.2243256056$

$-1.2768939685$

$-1.7213265019$

$-3.9850190406$

$-2.5485244536$

$-1.5557790921$

$-0.5111220178$

$-2.8041635286$

$-0.8365856451$

$-0.7879337057$

$-0.5672866544$

$-0.4745747606$

$-1.0287624707$

$-0.2691476880$

$-0.0603723651$

$-0.2555983247$

$-2.3161780157$

0.7304790678

$-1.0762514468$

$-0.8658418169$

0.9224057070

0.7558357893

$-0.7565095830$

$-2.8178840952$

$-2.9270626701$

1.3395546354

0.9214385980

$-0.8723077255$

$-1.9081201054$ 


$\begin{array}{lr}\mathrm{H} & 3.2557015900 \\ \mathrm{C} & 3.8631169577 \\ \mathrm{C} & 2.4502051458 \\ \mathrm{H} & 0.7450324749 \\ \mathrm{H} & -2.9189025627 \\ \mathrm{C} & -1.9339046537 \\ \mathrm{H} & -0.8064846464 \\ \mathrm{H} & 5.5643869186 \\ \mathrm{C} & 5.3256076063 \\ \mathrm{H} & 4.7987547935 \\ \mathrm{H} & 4.7350283734 \\ \mathrm{C} & 3.5779527149 \\ \mathrm{H} & 2.2187806175 \\ \mathrm{H} & -2.3062096641 \\ \mathrm{H} & 6.3025692944 \\ \mathrm{H} & 4.2287672653\end{array}$

\section{3b complete system}

$\begin{array}{ll}\text { Ru } & -0.9474381384 \\ \mathrm{C} & -2.2567188596 \\ \mathrm{C} & -2.7605536279 \\ \mathrm{C} & -2.9776809240 \\ \mathrm{C} & -1.1527482251 \\ \mathrm{C} & -3.9351407898 \\ \mathrm{H} & -2.2234185108 \\ \mathrm{C} & -4.1631728648 \\ \mathrm{H} & -2.6138751901 \\ \mathrm{C} & 2.7646965466 \\ \mathrm{C} & 1.8787908116 \\ \mathrm{C} & -1.9772523406 \\ \mathrm{C} & -0.9191124460 \\ \mathrm{C} & -4.6481729722 \\ \mathrm{H} & -4.2992820640 \\ \mathrm{H} & -4.7031374261 \\ \mathrm{C} & 3.7437377935 \\ \mathrm{C} & 3.0935298877 \\ \mathrm{C} & 2.9555644456 \\ \mathrm{C} & 1.6612209021 \\ \mathrm{H} & -2.1359780805 \\ \mathrm{C} & -2.5725574405 \\ \mathrm{C} & -1.5063396834 \\ \mathrm{H} & -0.2685305656 \\ \mathrm{H} & -5.5697462082 \\ \mathrm{H} & 3.5009590586 \\ \mathrm{C} & 4.9989456205 \\ \mathrm{C} & 4.3489779891 \\ \mathrm{H} & 2.3755872529 \\ \mathrm{H} & 3.1316321957\end{array}$

1.0873056245

2.9202701428

3.7060367567

2.4752285611

4.5417070788

4.3402877021

3.8559766954

$-1.1662028185$

$-2.6320329300$

$-3.9229466358$

3.0397186596

3.8695666706

4.4385288357

5.2839657853

$-3.0812574404$

4.7289929583
$-1.6378647290$

$-0.6937969230$

1.0945812198

1.5567694094

$-0.1341980365$

$-2.0399996662$

$-3.8162858240$

1.6933503990

0.1300447201

$-1.5139185062$

$-1.3315951880$

0.2889333891

1.8633041310

$-2.4288563758$

0.2842845452

0.4254123547

$\mathrm{E}(\mathrm{B} 3 \mathrm{LYP})=-1628.65405293553$
$-0.5064463246$
3.4691302150
$-1.8622889271$
0.8015823673
$-3.0330967182$
1.3992675839
$-1.3125941693$
$-0.2697891277$
1.2663994454
0.5677313349
$-3.6268911802$
0.9463268088
$-3.4742393713$
2.2320795780
$-1.9016709998$
$-0.7140176556$
$-0.4223898181$
$-0.7660440110$
$-1.9083168366$
1.1740178524
1.1979158203
2.1784219901
1.0607644110
1.4880548884
1.2380009243
$-0.8041980385$
$-3.0605518443$
$-0.1120516069$
$-4.5303833372$
1.4284908459
$-1.4504242892$
$-1.5424090999$
$-1.4759862611$
2.0881642831
$-2.9635660939$
0.2990140535
1.1973512897
0.1545760374
2.3502302429
2.0671109157
3.2480109805
1.8365571912
2.3049265241
0.5648779593
2.5552698248
$-1.4789370794$
0.8060901845
$-1.3447458084$
$-3.5181357119$
$-0.4612129946$
$-0.6755065465$
2.7777854937
$-2.0775529628$
2.1201136797
$-3.5749685045$
0.3455806697
$-3.2734298808$
$-0.4579544879$
0.3233583849
$-0.4636800804$ 


$\begin{array}{lrrr}\mathrm{C} & 3.7811695936 & 2.3113804971 & 0.0257062779 \\ \mathrm{C} & 2.4925748117 & 3.4603153289 & 1.7102951357 \\ \mathrm{H} & 0.8449430907 & 2.3603403085 & 2.5509059080 \\ \mathrm{H} & -3.2095151379 & 3.9369341472 & 1.1129074410 \\ \mathrm{C} & -2.3438465487 & 3.4393369312 & -0.7957284239 \\ \mathrm{H} & -1.3084479398 & 2.6967697736 & -2.5383005690 \\ \mathrm{H} & 5.7415529504 & -1.7273720407 & 2.8321373524 \\ \mathrm{C} & 5.3063784601 & -3.1328024186 & 1.2557998346 \\ \mathrm{H} & 4.5792070430 & -4.3845975939 & -0.3417178026 \\ \mathrm{H} & 4.6013559756 & 2.2919811902 & -0.6869009252 \\ \mathrm{C} & 3.5548380927 & 3.4469606316 & 0.8045719358 \\ \mathrm{H} & 2.3118108444 & 4.3381915235 & 2.3244577068 \\ \mathrm{H} & -2.8046907596 & 4.2728923662 & -1.3184986063 \\ \mathrm{H} & 6.2858273530 & -3.6015160674 & 1.2913845228 \\ \mathrm{H} & 4.2017348813 & 4.3144696667 & 0.7071186695 \\ \mathrm{H} & -1.5333853120 & -1.7044615945 & 4.3805778359 \\ \mathrm{C} & 0.2398320272 & -2.1039719230 & 1.2225370311 \\ \mathrm{C} & -0.9511728695 & -1.2919968563 & 1.2700825833 \\ \mathrm{C} & -0.4597079464 & 0.0976963392 & 1.2128222867 \\ \mathrm{C} & 0.9892407275 & 0.0278100106 & 1.1512205658 \\ \mathrm{C} & 1.4084241950 & -1.3221342925 & 1.1285716786 \\ \mathrm{C} & -0.9116541597 & 0.6094830237 & 5.0238961815 \\ \mathrm{O} & -0.8996898239 & 1.2924818894 & 5.9548266666 \\ \mathrm{C} & 0.7161641683 & -1.8867113869 & 4.4242868935 \\ \mathrm{O} & 1.2252415922 & -0.8227975261 & 3.9785619027 \\ \mathrm{H} & 0.5105989544 & -2.0113953877 & 5.4966212535 \\ \mathrm{H} & 0.6979842250 & -2.8013979759 & 3.8141256347 \\ \mathrm{C} & -2.8003509564 & -0.2106484542 & 3.2555188980 \\ \mathrm{O} & -3.9415116608 & -0.0646029825 & 3.2117875598 \\ \mathrm{O} & 0.2075262997 & -3.4478762568 & 1.3006101216 \\ \mathrm{H} & 1.1242181062 & -3.7728967341 & 1.2758996920\end{array}$

\section{4b complete system $\quad \mathrm{E}(\mathrm{B} 3 \mathrm{~L} Y \mathrm{P})=-1628.66502499282$}

$\begin{array}{lccc}\mathrm{Ru} & -0.9325948818 & -0.3261969649 & 1.6665791054 \\ \mathrm{H} & -0.9455002932 & -1.7962808391 & 3.0451093461 \\ \mathrm{C} & 0.2265470897 & -1.6730112927 & -0.8312811406 \\ \mathrm{C} & -0.9251403019 & -0.8788177404 & -0.6600334263 \\ \mathrm{C} & -0.4202177030 & 0.4740011999 & -0.2957379359 \\ \mathrm{C} & 1.0914067541 & 0.3718075018 & -0.3660742451 \\ \mathrm{C} & 1.4484199907 & -0.9189405356 & -0.6841625549 \\ \mathrm{C} & -0.6542736092 & 1.0279406193 & 2.9388535672 \\ \mathrm{O} & -0.4564718558 & 1.9176730520 & 3.6538143457 \\ \mathrm{C} & 0.1911441688 & -2.0894641314 & 2.9804954519 \\ \mathrm{O} & 0.8529789470 & -1.3226633000 & 2.0584966081 \\ \mathrm{H} & 0.5303008935 & -1.9651817819 & 4.0240644358 \\ \mathrm{H} & 0.1333330516 & -3.1623514421 & 2.7295465092 \\ \mathrm{C} & -2.7880995177 & 0.1253736292 & 1.5450878596 \\ \mathrm{O} & -3.9264176096 & 0.3192463192 & 1.5755103607\end{array}$




$\begin{array}{lrrr}\mathrm{O} & 0.2079635462 & -2.9793868273 & -1.0931187327 \\ \mathrm{H} & 1.1284335533 & -3.2972854972 & -1.1262544872 \\ \mathrm{C} & -2.2783262598 & -1.3135094849 & -1.1191381304 \\ \mathrm{C} & -2.7333896750 & -2.6124213517 & -0.8155777742 \\ \mathrm{C} & -3.1207913787 & -0.4910159819 & -1.8865755335 \\ \mathrm{C} & -0.9946478977 & 1.7394922899 & -0.8900319396 \\ \mathrm{C} & -3.9725421800 & -3.0667216749 & -1.2584928506 \\ \mathrm{H} & -2.1066438304 & -3.2698989690 & -0.2232002144 \\ \mathrm{C} & -4.3664740302 & -0.9461236396 & -2.3207743735 \\ \mathrm{H} & -2.8040910989 & 0.5077102800 & -2.1551523581 \\ \mathrm{C} & 2.7643469584 & -1.5963725313 & -0.7266086291 \\ \mathrm{C} & 2.0056169678 & 1.5051009280 & -0.1642942766 \\ \mathrm{C} & -1.7800251005 & 2.6618687426 & -0.1911835068 \\ \mathrm{C} & -0.6848465435 & 2.0238209769 & -2.2335628591 \\ \mathrm{C} & -4.8009156574 & -2.2336750277 & -2.0123940172 \\ \mathrm{H} & -4.2945799279 & -4.0737216106 & -1.0060506463 \\ \mathrm{H} & -4.9963901079 & -0.2854051589 & -2.9107294627 \\ \mathrm{C} & 3.5961511606 & -1.6512792903 & 0.4057681876 \\ \mathrm{C} & 3.1741417678 & -2.2565148588 & -1.9001254476 \\ \mathrm{C} & 3.2092214958 & 1.5769304681 & -0.8935970213 \\ \mathrm{C} & 1.6972394327 & 2.5677863879 & 0.7043025071 \\ \mathrm{H} & -2.0103306765 & 2.4853600142 & 0.8526907210 \\ \mathrm{C} & -2.2636889181 & 3.8150564602 & -0.8153379633 \\ \mathrm{H} & -1.1636950074 & 3.1717954069 & -2.8584993652 \\ \mathrm{H} & 4.4281065563 & 4.5450196712 & 0.2379560958 \\ \mathrm{H} & -0.0650953588 & 1.3267715292 & -2.7918342681 \\ \mathrm{H} & -5.7709952622 & -2.5845693905 & -2.3536048335 \\ \mathrm{H} & 3.2527541818 & -1.1855654622 & 1.3227550182 \\ \mathrm{C} & 4.8135259918 & -2.3253329106 & 0.3500860880 \\ \mathrm{C} & 4.3920233040 & -2.9404995097 & -1.9466072393 \\ \mathrm{H} & 2.5508725744 & -2.2026484829 & -2.7904191248 \\ \mathrm{H} & 3.4579098649 & 0.7874646244 & -1.5932800171 \\ \mathrm{C} & 4.0705284805 & 2.6605992015 & -0.7515020418 \\ \mathrm{C} & 2.5642413973 & 3.6467251406 & 0.8515968622 \\ \mathrm{H} & 0.7794419826 & 2.5427409186 & 1.2758167115 \\ \mathrm{H} & -2.8758173624 & 4.5122023884 & -0.2495586681 \\ \mathrm{H} & -1.9622586318 & 4.0732885157 & -2.1502229088 \\ \mathrm{H} & -0.9138381163 & 3.3631989397 & -3.8987882402 \\ \mathrm{H} & 5.2156597813 & -2.9713700804 & -0.8230352708 \\ \mathrm{H} & 4.6960948169 & -3.4390818280 & -2.8629056311 \\ \mathrm{H} & 2.3063478785 & 4.4500100364 & 1.5360949915 \\ \mathrm{H} & -1643741143 & -3.4998546316 & -0.8571870875 \\ \mathrm{H} & & & -1.3325121926 \\ \mathrm{H} & & & \end{array}$




\begin{tabular}{|c|c|c|c|}
\hline $\mathrm{Ru}$ & 0.0206907296 & -0.7346008439 & 1.1873682622 \\
\hline $\mathrm{H}$ & 1.2940620282 & -3.5216405397 & 2.8446400825 \\
\hline $\mathrm{C}$ & 0.0266141695 & -1.4343547678 & -1.1226943325 \\
\hline $\mathrm{C}$ & -1.1888565384 & -0.6813338097 & -0.7464967868 \\
\hline $\mathrm{C}$ & -0.7989669078 & 0.6627109139 & -0.4005707412 \\
\hline $\mathrm{C}$ & 1.1747641891 & -0.5572740954 & -0.7798310559 \\
\hline $\mathrm{C}$ & 1.3524355471 & -0.2921459475 & 2.4906131717 \\
\hline $\mathrm{O}$ & 2.1728267673 & 0.0286151296 & 3.2398043530 \\
\hline $\mathrm{C}$ & 1.3384020708 & -3.6878797981 & 1.7672194502 \\
\hline $\mathrm{O}$ & 0.2131273763 & -3.0159268876 & 1.1812022651 \\
\hline $\mathrm{H}$ & 2.2843318981 & -3.2998189838 & 1.3741792582 \\
\hline $\mathrm{H}$ & 1.2702759746 & -4.7629075893 & 1.5694346155 \\
\hline $\mathrm{C}$ & -1.3655171872 & -0.6392495664 & 2.5065771788 \\
\hline $\mathrm{O}$ & -2.2253852402 & -0.5650739840 & 3.2750 \\
\hline $\mathrm{O}$ & 0.078 & -2.65 & $-1.42 \varepsilon$ \\
\hline $\mathrm{H}$ & 0.2078701536 & -3.178 & 0.1967 \\
\hline $\mathrm{C}$ & -2.5551392185 & -1.1886228204 & -1.0235613459 \\
\hline $\mathrm{C}$ & -2.9706793091 & -2.436 & -0.5327 \\
\hline $\mathrm{C}$ & -3.43 & -0.4 & -1.8 \\
\hline $\mathrm{C}$ & 0.6531780469 & 0.734 & -0.41 \\
\hline $\mathrm{C}$ & -1.7035652219 & 1038940 & -0.3109699907 \\
\hline $\mathrm{C}$ & -4.2348477093 & -2.935 & -0.8400 \\
\hline $\mathrm{H}$ & -2.2998904571 & -3.01 & 0.0948402663 \\
\hline $\mathrm{C}$ & -4.6981977480 & -0.9668412356 & -2.1522391480 \\
\hline $\mathrm{H}$ & -3.1250555766 & 0.4928615011 & -2.2519137423 \\
\hline $\mathrm{C}$ & 2.5878976 & -0.9393020 & -1.01 \\
\hline $\mathrm{C}$ & 1.4248702866 & 1.998 & -0.2761350279 \\
\hline $\mathrm{C}$ & -2.8034442181 & 1.88573085 & 0.5583080048 \\
\hline $\mathrm{C}$ & -1.4967971787 & 2.9223455276 & -1.1862037649 \\
\hline $\mathrm{C}$ & -5.1047525684 & -2.2023922 & -1.64 \\
\hline $\mathrm{H}$ & -4.5407468448 & -3.901 & 12485 \\
\hline $\mathrm{H}$ & -5.3644251924 & -0.3907108369 & -2.78 \\
\hline $\mathrm{C}$ & 595555 & -0.417 & -0.24 \\
\hline $\mathrm{C}$ & 2.9003659368 & -1.836 & -2.0481720215 \\
\hline $\mathrm{C}$ & 2.2761638942 & 2.3943789839 & -1.3200030286 \\
\hline $\mathrm{C}$ & 1.2735244179 & 2.8470617766 & 0.8299992044 \\
\hline $\mathrm{H}$ & -2.9869221840 & 1.060 & 1.23604 \\
\hline $\mathrm{C}$ & -3.6657813695 & 2.9802744283 & 0.5602928867 \\
\hline $\mathrm{C}$ & -2.3645908727 & 4.0139078424 & -1.1869456105 \\
\hline $\mathrm{H}$ & -0.6564421229 & 2.9050200408 & -1.8722964509 \\
\hline $\mathrm{H}$ & -6.0906552429 & -2.5925742092 & -1.8862646657 \\
\hline $\mathrm{H}$ & 3.4331720227 & 0.2697336315 & 0.5700713510 \\
\hline $\mathrm{C}$ & 4.9644525035 & -0.7701287592 & -0.5081789359 \\
\hline $\mathrm{C}$ & 4.2243756090 & -2.1856126897 & -2.3123267100 \\
\hline $\mathrm{H}$ & 2.0974482532 & -2.2651676531 & -2.6359922379 \\
\hline $\mathrm{H}$ & 2.4027011393 & 1.7474679232 & -2.1823419087 \\
\hline C & 2.9541245693 & 3.6112402464 & -1.2595319984 \\
\hline $\mathrm{C}$ & 1.9557797682 & 4.0606177440 & 0.8901734322 \\
\hline $\mathrm{H}$ & 0.6175239143 & 2.5505241282 & 1.6423810012 \\
\hline $\mathrm{H}$ & -4.5089048721 & 2.9963733675 & 1.2452894287 \\
\hline
\end{tabular}




$\begin{array}{lr}\mathrm{C} & -3.4501331095 \\ \mathrm{H} & -2.1888222399 \\ \mathrm{H} & 5.7627384039 \\ \mathrm{C} & 5.2622021857 \\ \mathrm{H} & 4.4426046104 \\ \mathrm{H} & 3.6063014816 \\ \mathrm{C} & 2.7972932797 \\ \mathrm{H} & 1.8289429334 \\ \mathrm{H} & -4.1242166094 \\ \mathrm{H} & 6.2930979830 \\ \mathrm{H} & 3.3281809517\end{array}$

\section{b complete system}

$\begin{array}{lr}\mathrm{Ru} & 0.0048732952 \\ \mathrm{C} & -1.2118184238 \\ \mathrm{C} & -0.7282141878 \\ \mathrm{C} & 0.7443318991 \\ \mathrm{C} & 1.1774565580 \\ \mathrm{C} & -0.0382340984 \\ \mathrm{C} & 1.3928902609 \\ \mathrm{O} & 2.2473100829 \\ \mathrm{O} & -0.0718085675 \\ \mathrm{C} & -1.3604267749 \\ \mathrm{O} & -2.2092026592 \\ \mathrm{C} & -2.5992074327 \\ \mathrm{C} & -2.9995990122 \\ \mathrm{H} & -2.2923433786 \\ \mathrm{C} & -4.2855405287 \\ \mathrm{H} & -4.5772854922 \\ \mathrm{C} & -5.1925426097 \\ \mathrm{H} & -6.1945343106 \\ \mathrm{C} & -4.8006421181 \\ \mathrm{H} & -5.4946157886 \\ \mathrm{C} & -3.5162908803 \\ \mathrm{H} & -3.2225079573 \\ \mathrm{C} & -1.5368073621 \\ \mathrm{C} & -2.5901931804 \\ \mathrm{H} & -2.8033607258 \\ \mathrm{C} & -3.3684695640 \\ \mathrm{H} & -4.1774732641 \\ \mathrm{C} & -3.1144883661 \\ \mathrm{H} & -3.7250066510 \\ \mathrm{C} & -2.0724797995 \\ \mathrm{H} & -1.8672116839 \\ \mathrm{C} & -1.2859188554 \\ \mathrm{H} & -0.4774765020 \\ \mathrm{C} & 1.5906450335 \\ \mathrm{C} & 2.4862581585 \\ & \end{array}$

4.0484154522

4.8380785425

$-0.3524784177$

$-1.6546342109$

$-2.8773752278$

3.9034966788

4.4478314355

4.7046197606

4.9005966288

$-1.9280929181$

5.3945516843
$-0.3117946099$

$-1.8727881597$

0.0993302813

$-1.5470163870$

$-3.1215820277$

$-2.0779067393$

$-0.1541148045$

1.7558936547

$-0.3095716641$

$-1.7547976610$

$-0.1055753890$

$\mathrm{E}(\mathrm{B} 3 \mathrm{LYP})=-1512.97543323668$

$-0.7562431634$

$-0.8661405680$

0.4619980140

0.4341745826

$-0.9065750759$

$-1.7529070213$

$-0.3916670553$

$-0.1001541632$

$-2.9684431933$

$-0.4961413665$

$-0.2995385411$

$-1.3375949632$

$-2.5851170412$

$-3.1794747229$

$-3.0683395167$

$-4.0328453802$

$-2.3234745783$

$-2.7025907943$

$-1.0926237975$

$-0.5107907960$

$-0.6032339965$

0.3483515994

1.7097863734

1.9467098660

1.2295191639

3.0972620687

3.2657536890

4.0257560787

4.9204039363

3.7986367944

4.5138692370

2.6528572769

2.4845357845

1.6536232478

1.8445333547
1.3686880228

$-0.5620438926$

$-0.3833368266$

$-0.4088578790$

$-0.6020801471$

$-0.8307624187$

2.6431603990

3.3638677355

$-1.0267606957$

2.6868067277

3.4462214313

$-0.7728016038$

$-0.2629030554$

0.3047074024

$-0.4943002895$

$-0.0878323632$

$-1.2493434545$

$-1.4313912981$

$-1.7769769376$

$-2.3776346810$

$-1.5438182656$

$-1.9732780888$

$-0.4120574009$

0.4833892529

1.2673233185

0.3730542851

1.0781973243

$-0.6377666066$

$-0.7236844348$

$-1.5373223578$

$-2.3288952479$

$-1.4237209456$

$-2.1280984576$

$-0.4225901669$

$-1.4876498919$ 


$\begin{array}{lrrr}\mathrm{H} & 2.5831726337 & 1.0776159061 & -2.2500695630 \\ \mathrm{C} & 3.2456431935 & 3.0103108570 & -1.5728165058 \\ \mathrm{H} & 3.9312204706 & 3.1453280294 & -2.4047399711 \\ \mathrm{C} & 3.1282975328 & 3.9964451924 & -0.5922019895 \\ \mathrm{H} & 3.7238096681 & 4.9029320572 & -0.6561301564 \\ \mathrm{C} & 2.2436048463 & 3.8125974066 & 0.4718682090 \\ \mathrm{H} & 2.1486514678 & 4.5750027459 & 1.2398497546 \\ \mathrm{C} & 1.4760883128 & 2.6523665585 & 0.5554754005 \\ \mathrm{H} & 0.7866700398 & 2.5123803995 & 1.3820042781 \\ \mathrm{C} & 2.5563285467 & -1.4281055647 & -0.7358981777 \\ \mathrm{C} & 3.6356662596 & -0.8863174319 & -0.0133935091 \\ \mathrm{H} & 3.4664747643 & -0.0731332064 & 0.6830983240 \\ \mathrm{C} & 4.9250793034 & -1.3841445877 & -0.1760950090 \\ \mathrm{H} & 5.7417755046 & -0.9519069558 & 0.3955031586 \\ \mathrm{C} & 5.1675688282 & -2.4355622049 & -1.0629672967 \\ \mathrm{H} & 6.1745643016 & -2.8243076347 & -1.1877251565 \\ \mathrm{C} & 4.1065181861 & -2.9857787803 & -1.7811197464 \\ \mathrm{H} & 4.2822734196 & -3.8073453273 & -2.4701677876 \\ \mathrm{C} & 2.8123211159 & -2.4930983922 & -1.6194608798 \\ \mathrm{H} & 1.9906838722 & -2.9388771528 & -2.1661575821\end{array}$

\section{ts1d complete system}

$\begin{array}{lr}\mathrm{Ru} & 0.0130880924 \\ \mathrm{H} & 0.4053202712 \\ \mathrm{C} & -1.1781826545 \\ \mathrm{C} & -0.8165973053 \\ \mathrm{C} & 0.6223090141 \\ \mathrm{C} & 1.1717683913 \\ \mathrm{C} & 0.0467897860 \\ \mathrm{C} & 1.2789400024 \\ \mathrm{O} & 2.0653189241 \\ \mathrm{O} & 0.0995354651 \\ \mathrm{H} & 0.6601715023 \\ \mathrm{C} & 0.6729515709 \\ \mathrm{O} & 1.2538480905 \\ \mathrm{H} & 1.2778871900 \\ \mathrm{H} & -0.3795476538 \\ \mathrm{C} & -1.4073758124 \\ \mathrm{O} & -2.2914058431 \\ \mathrm{C} & -2.5213375386 \\ \mathrm{C} & -2.9204941023 \\ \mathrm{H} & -2.2568215488 \\ \mathrm{C} & -4.1504503446 \\ \mathrm{H} & -4.4405767889 \\ \mathrm{C} & -5.0046483195 \\ \mathrm{H} & -5.9634839786 \\ \mathrm{C} & -4.6157232076 \\ \mathrm{H} & -5.2683571872\end{array}$

$E(B 3 L Y P)=-1628.69504038493$

$\begin{array}{cc}-0.6115834893 & 1.3077527018 \\ -2.2144268208 & 1.7635292377 \\ -0.7084235414 & -0.6711074057 \\ 0.6765135612 & -0.4118132095 \\ 0.7708209048 & -0.4157662868 \\ -0.5470284906 & -0.6757401248 \\ -1.4384631499 & -0.9026687614 \\ -0.0360299987 & 2.6135583779 \\ 0.3242983413 & 3.3792985430 \\ -2.7090489472 & -1.2229138356 \\ -3.3110622736 & -0.5177860342 \\ -3.6183378726 & 1.6453496445 \\ -3.8952370732 & 0.5456502246 \\ -3.6215692916 & 2.5641487805 \\ -3.9137060204 & 1.8018487999 \\ -0.6025531529 & 2.5809562046 \\ -0.6085295231 & 3.3255269161 \\ -1.2612906287 & -0.9763272766 \\ -2.5120202223 & -0.4808696024 \\ -3.0585716004 & 0.1796546627 \\ -3.0599336637 & -0.8375089959 \\ -4.0299472582 & -0.4433600776 \\ -2.3699569662 & -1.6992537067 \\ -2.7982581469 & -1.9779855161 \\ -1.1301322251 & -2.2052464287 \\ -0.5876122087 & -2.8837451124\end{array}$




$\begin{array}{lrrr}\mathrm{C} & -3.3842969733 & -0.5809574447 & -1.8501832711 \\ \mathrm{H} & -3.0879339722 & 0.3777941041 & -2.2614486456 \\ \mathrm{C} & -1.7583144194 & 1.8265062255 & -0.3710897564 \\ \mathrm{C} & -2.8816110261 & 1.8413882878 & 0.4702641890 \\ \mathrm{H} & -3.0567996870 & 1.0135787845 & 1.1481862911 \\ \mathrm{C} & -3.7742981937 & 2.9104606910 & 0.4463739040 \\ \mathrm{H} & -4.6347409443 & 2.9042784260 & 1.1095559555 \\ \mathrm{C} & -3.5649116411 & 3.9836465267 & -0.4215641260 \\ \mathrm{H} & -4.2609765543 & 4.8176852236 & -0.4374712525 \\ \mathrm{C} & -2.4570254395 & 3.9774635124 & -1.2685865753 \\ \mathrm{H} & -2.2856514784 & 4.8052743238 & -1.9510702964 \\ \mathrm{C} & -1.5615034681 & 2.9087386163 & -1.2448093821 \\ \mathrm{H} & -0.7043483133 & 2.9116856535 & -1.9103656481 \\ \mathrm{C} & 1.4066735938 & 2.0321169347 & -0.3323791955 \\ \mathrm{C} & 2.3028417753 & 2.3506246622 & -1.3654041831 \\ \mathrm{H} & 2.4391226305 & 1.6584216113 & -2.1899729882 \\ \mathrm{C} & 3.0195320783 & 3.5466249779 & -1.3400343765 \\ \mathrm{H} & 3.7092812987 & 3.7756667287 & -2.1475885455 \\ \mathrm{C} & 2.8562627746 & 4.4408797291 & -0.2819254370 \\ \mathrm{H} & 3.4198556191 & 5.3694613599 & -0.2590454363 \\ \mathrm{C} & 1.9664809053 & 4.1342237110 & 0.7489721276 \\ \mathrm{H} & 1.8338337382 & 4.8228244137 & 1.5786836607 \\ \mathrm{C} & 1.2466330267 & 2.9415725224 & 0.7236071536 \\ \mathrm{H} & 0.5571408328 & 2.7069520305 & 1.5279699645 \\ \mathrm{C} & 2.5883917179 & -0.9134186077 & -0.9241939183 \\ \mathrm{C} & 3.6417102201 & -0.4307892962 & -0.1306155938 \\ \mathrm{H} & 3.4275838534 & 0.2015976257 & 0.7238265665 \\ \mathrm{C} & 4.9628216967 & -0.7562415102 & -0.4268806012 \\ \mathrm{H} & 5.7626571807 & -0.3738533124 & 0.2012376156 \\ \mathrm{C} & 5.2591445099 & -1.5725127115 & -1.5202573069 \\ \mathrm{H} & 6.2904414334 & -1.8269705963 & -1.7489714767 \\ \mathrm{C} & 4.2218183928 & -2.0629303455 & -2.3124651278 \\ \mathrm{H} & 4.4401688190 & -2.7026485070 & -3.1631668925 \\ \mathrm{C} & 2.8978475301 & -1.7396035929 & -2.0181318042 \\ \mathrm{H} & 2.0961720221 & -2.1323931178 & -2.6339858291 \\ & & & \end{array}$

3. Comparison between B3LYP, MP2 and CCSD(T) calculations for the model system

Table S1. Relative energies in gas phase $(\mathrm{kcal} / \mathrm{mol})$ calculated at B3LYP, MP2 and CCSD(T) levels. Geometries optimized at B3LYP level. 


\begin{tabular}{|c|c|c|c|c|}
\hline & Structure & B3LYP & MP2 & $\overline{C C C S D(T)}$ \\
\hline Reactants & $\mathrm{a} 1+\mathrm{H}_{2} \mathrm{CO}$ & 0.0 & 0.0 & 0.0 \\
\hline \multirow{7}{*}{ CO leaving mechanism } & $1 \mathrm{a}$ & 51.2 & 65.1 & 53.3 \\
\hline & 2a & 23.8 & 20.2 & 9.3 \\
\hline & ts1a & 31.7 & 35.5 & 18.5 \\
\hline & 3a & 29.5 & 34.8 & 14.7 \\
\hline & $4 a 2$ & -3.9 & -1.4 & -7.9 \\
\hline & ts2a & -0.6 & 1.5 & -4.0 \\
\hline & $5 a$ & -23.6 & -30.5 & -28.2 \\
\hline \multirow{8}{*}{$\mathrm{CpOH}_{\text {ring slippage mechanism }}^{a}$} & $1 \mathbf{b}$ & -10.6 & -12.6 & -11.7 \\
\hline & ts1b & 19.3 & 24.9 & 19.8 \\
\hline & $2 \mathbf{b}$ & 18.0 & 26.9 & 20.5 \\
\hline & $3 \mathbf{b}$ & 22.2 & 29.3 & 24.3 \\
\hline & ts2b & 23.7 & 31.9 & 26.7 \\
\hline & 4b & 17.8 & 25.9 & 19.3 \\
\hline & ts3b & 22.0 & 26.6 & 20.5 \\
\hline & $5 a$ & -23.8 & -30.6 & -28.1 \\
\hline Concerted inner sphere mechanism $^{a}$ & ts1c & 25.4 & 30.0 & 23.3 \\
\hline \multirow[t]{2}{*}{ Concerted outer sphere mechanism $^{a}$} & ts1d & -1.5 & -0.5 & 2.7 \\
\hline & 2c & -12.9 & -18.6 & -13.2 \\
\hline Products $^{a}$ & $\mathrm{~b}+\mathrm{CH}_{3} \mathrm{OH}$ & -0.2 & -2.4 & 3.2 \\
\hline
\end{tabular}

${ }^{a}$ These energies are referred to $\mathbf{a} 2+\mathbf{H}_{\mathbf{2}} \mathbf{C O}$ 